\title{
Hybrid Swarm Intelligence Energy Efficient Clustered Routing Algorithm for Wireless Sensor Networks
}

\author{
Rajeev Kumar ${ }^{1}$ and Dilip Kumar ${ }^{2}$ \\ ${ }^{1}$ Punjab Technical University, Jalandhar 144001, India \\ ${ }^{2}$ Department of Electronics and Communication Engineering, S.L.I.E.T., Longowal 148106, India \\ Correspondence should be addressed to Dilip Kumar; dilip.k78@gmail.com
}

Received 3 July 2015; Revised 19 August 2015; Accepted 26 August 2015

Academic Editor: Pietro Siciliano

Copyright ( 2016 R. Kumar and D. Kumar. This is an open access article distributed under the Creative Commons Attribution License, which permits unrestricted use, distribution, and reproduction in any medium, provided the original work is properly cited.

Currently, wireless sensor networks (WSNs) are used in many applications, namely, environment monitoring, disaster management, industrial automation, and medical electronics. Sensor nodes carry many limitations like low battery life, small memory space, and limited computing capability. To create a wireless sensor network more energy efficient, swarm intelligence technique has been applied to resolve many optimization issues in WSNs. In many existing clustering techniques an artificial bee colony (ABC) algorithm is utilized to collect information from the field periodically. Nevertheless, in the event based applications, an ant colony optimization (ACO) is a good solution to enhance the network lifespan. In this paper, we combine both algorithms (i.e., ABC and $\mathrm{ACO}$ ) and propose a new hybrid ABCACO algorithm to solve a Nondeterministic Polynomial (NP) hard and finite problem of WSNs. ABCACO algorithm is divided into three main parts: (i) selection of optimal number of subregions and further subregion parts, (ii) cluster head selection using $\mathrm{ABC}$ algorithm, and (iii) efficient data transmission using ACO algorithm. We use a hierarchical clustering technique for data transmission; the data is transmitted from member nodes to the subcluster heads and then from subcluster heads to the elected cluster heads based on some threshold value. Cluster heads use an ACO algorithm to discover the best route for data transmission to the base station (BS). The proposed approach is very useful in designing the framework for forest fire detection and monitoring. The simulation results show that the ABCACO algorithm enhances the stability period by $60 \%$ and also improves the goodput by $31 \%$ against LEACH and WSNCABC, respectively.

\section{Introduction}

Wireless sensor networks (WSNs) consist of various sensor nodes, which have the capability to sense, process, compute, and communicate [1]. A large number of sensor nodes and their different applications induce various hindrances during implementation in sensor networks. Other than implementation, certain areas, namely, accessing of information, data collection, and storage, are still a dilemma. Various specifications of sensor nodes like their size, computational ability, cost, hardware constraint, energy efficiency, and other design parameters make the implementation of the sensor network a difficult task. In present scenario optimization issues become more tedious. An energy specification of sensor networks becomes intolerable in a huge environment which in turn demands a concern related to various optimization parameters. That is why we formulated to assess the potency of various evolutionary algorithms to enhance the network lifespan with energy as a constraint duly concentrating on the various transmission strategies [2]. During a couple of years, a number of evolutionary algorithms have been introduced for evaluation of problems to get the best possible result out of the various solutions. Many of the proposed algorithms concentrate on the principle of the population based heuristic search methods, for solving general optimization problems. A swarm intelligent algorithm works on the principle behavior of flying birds in order to get to the resources being implemented in LEACH [3] protocol. In these protocols, the swarm intelligent algorithm is implemented to form various clusters which in turn help in defining their respective $\mathrm{CHs}$ while designing a WSN with the same conditions as in real time network. The swarm based algorithm produces desirable 
results. Network lifespan can be further enhanced by implementing various best possible route detection techniques. Artificial bee colony (ABC) algorithm is a new upcoming approach whose working totally resembles that of swarm based optimization techniques while monitoring the clusters in WSN.

The most unavoidable hindrances faced in the sensor networks are about getting the best possible routes for data transmission up to the BS. In general WSN node operates in a multinode pattern; immense algorithms have been introduced for routing data to reach the destination. In [4] some notable routing techniques in WSN desire to use ant as a moving agent while discovering the route in order to discover the best possible path for data transmission in WSN. In this research, we prioritize the multiple nodes grounded in their respective power, competency, and there approaching nature to route the data through the best path. In recent years, a new picture by applying routing based protocols on the movement of the swarms in order to depict the best possible path for delivering the acquired data through the selected path was introduced. Algorithm working with nodes should be more energy saver as well as fast enough to acquire and transmit the data. Ant colony optimization (ACO), designed in such a way so that ants behaviors for finding food sources can be depicted easily, acts as a valuable solution for different route detection, although this technique is not favourable in governing applications which needs timely data transfer [5].

For upgrading the execution of the network, clustering mechanisms have been connected to networks with hierarchical structures while minimizing the fundamental energy utilization $[6,7]$. Clustering is a technique to group number of nodes in areas in a densely employed wide-scale network [8]. One of the nodes will be elected as a cluster head $(\mathrm{CH})$ among other nodes called cluster members of their respective cluster. In clustering, randomly deployed nodes join in carrying out the job. Here, the signal to the nodes is advertised with nodes. Signals are received by all sensor nodes from distinct $\mathrm{CH}$ and nodes give response with their node-id to $\mathrm{CH}$ with strength and this formation of clusters takes place. Without $\mathrm{CH}$, for intracluster routing, a proactive strategy is utilized and for intercluster routing, a reactive strategy is utilized. Clustering has various challenges in deployment such as ensuring connectivity between nodes, determining optimal cluster sizes $[9,10]$, and optimizing clustering structures dynamically on the basis of the status of cluster members. For a hierarchical routing or network administration, convention can be better executed with CHs. In [11, 12], the authors have studied the influence of cluster based hierarchical routing that is a quite famous approach with some benefits related to well organized communication and scalability. For attaining power efficient routing, the concept of hierarchical routing is used. Using two-tier clusters for sending information to BS has the profit of shorter transmit separations for nodes. Only some nodes are essential to send data to BS at far distances and it is valid in those networks where there is a high density of nods. Through hierarchical level cluster can communicate with other clusters or with external network at the same time [13]. In this paper, we have introduced threelevel hierarchy. The first level is known as $\mathrm{CH}$, the second level is known as subcluster head (SCH), and the third level is denoted by member node. Partial local computation is introduced in each and every $\mathrm{SCH}$ at the second stage and the local computation at $\mathrm{CH}$ where information is sent to BS directly. This approach is used as regions and subregions and significant progress is obtained. The objectives of the subregions and subregion parts are formulated as in

$$
\operatorname{Maximum}\left(T_{\mathrm{SSC}}\right)=\operatorname{Minimum} \sum_{i, j \in \mathrm{Tee}} E_{\mathrm{Tee}}
$$

where $T_{\mathrm{SSC}}$ is the work time of the square shaped cluster. $E_{\mathrm{Tee}}$ is the total energy expenditure in a cluster.

The rest of the work is done in seven sections. In Section 2, we discuss the related work. In Section 3, we define the radio model for wireless sensor network. Section 4 describes the squared optimization problem for $\mathrm{CH}$ and $\mathrm{SCH}$. Section 5 exhibits the details of ABC, ACO, and the proposed approach ABCACO. In Section 6, the performances of ABCACO approach are evaluated via a number of experiments and we compare its simulation results with both WSNCABC and LEACH. Finally, Section 7 concludes the paper and suggests the further work to be carried out.

\section{Related Works}

In literature, various protocols have been considered with the idea of enhancing the lifespan of sensor network by the appropriation of cluster based sensor network structural engineering. A well-known protocol known as LEACH (Low Energy Adaptive Clustering Hierarchy) [3] is based on distributed clustering approach. It prolongs the network lifespan by reducing the power dissipation. No wastage of power for electing a node as a $\mathrm{CH}$ is the advantage of $\mathrm{LEACH}$ protocol. The $\mathrm{CH}$ selection process is primarily based upon the inducted energy level of each sensor node that is the disadvantage of LEACH. So a sensor node that has low power has more chances of becoming a $\mathrm{CH}$ than the one that has more power which leads to the dead state of the entire cluster. The second disadvantage is the presence of more overheads in the development of the dynamic cluster. In [9], the results have demonstrated that LEACH-C outperforms LEACH because of the improvement in cluster formation by the BS. Furthermore, the fraction of $\mathrm{CH}$ in each iteration of LEACH-C is equivalent to the craved optimum number. An additional clustering algorithm that enhances the lifespan of network has been presented in [14] known as HEED (Hybrid Energy Efficient Distributed Clustering). The $\mathrm{CH}$ election process in HEED is intentionally based upon the parameters, that is, residual energy of the sensor nodes, and in addition to this cost of communication within the clusters is also considered by the authors for increasing power efficiency and further enhancing lifespan of network. This protocol maintains the $\mathrm{CH}$ for a fixed number of cycles. Related to LEACH, significant overhead is imposed by the sensor network by clustering in each round. Noticeable power dissipation is imposed by this overhead that leads to decrease in lifespan of the network. As HEED requires various iterations for the formation of clusters, it suffers from a subsequent 
overhead as at each iteration the broadcasting of a lot of packets takes place. Power Efficient Gathering in Sensor Information Systems (PEGASIS) has been developed in [15] for prolonging the network lifespan. PEGASIS outperforms $\mathrm{LEACH}$ in terms of power dissipation for extending the network lifespan. It is not a clustering approach. It is a chain based protocol due to which all nodes interact with one another and can transmit data to a $\mathrm{CH}$. Sensor nodes die in arbitrary locations in PEGASIS as a selection of $\mathrm{CHs}$ has been done without concerning the overall lifespan of each sensor node. Delay in transmitting time bounds data over a chain of sensor nodes is the main disadvantage of PEGASIS. Optimal routing may not be present as it is a greedy algorithm that selects the shortest path rather than the optimal one and there is just simply $\mathrm{CH}$ that may turn out to be a bottleneck when a lot of data is gathered at this sensor node. In [4] it is suggested in the literature that various power conserving methods are presented that tend to prolong the lifespan of the network. The author explained a converged, power efficient cluster based protocol that is considered to prolong the lifespan of the network using a swarm intelligence mechanism, called artificial bee colony $(\mathrm{ABC})$. In [16] the authors introduce the EEABR protocol, which is in view of the ACO heuristic centred on the standard WSN requirements. They propose two redesigns in the AntNet routing algorithm for reducing the memory utilized as a piece of sensor nodes as well as dealing with the energy constraints of the routes discovered by the mobile agents. In this paper the author has presented the enacted energy level of sensor nodes and their routing distance with the ACO probability equation by adjusting the routing conventions in WSN. Likewise the algorithm considered data aggregation yet did not consider the adjusted utilization of energy in the entire networks. The protocol exhibited in [17] introduced improved information driven routing protocol of WSN based upon the ant including the search ant, so as to provide past data to the neighbourhood ants. It described a "retry" principle so that the protocol does not come to a halt. In this the communication cost known as the Euclidean distance between the two vertexes is considered such that they can correspond with one another specifically. Taking into account this expense, it builds a weighted graph by consuming three types of ants, namely, chasing ants, foragers, and trailing ants. Chasing ants are specifically used to make the forward ants searching the sink (the destination node) in a scalable manner. In the proposed protocol, the source nodes were only $2.5 \%$ of the total nodes. No framework lifespan utilizing this algorithm was as a part of the paper. In [4] the authors have met the prerequisite of WSN by adjusting ant colony and presenting information on a chip based routing component for streamlining proficiency; however it has been connected to the planar routing. In [18] the authors are concerned with the problem of multiagent path planning in discrete-time pursuit-evasion games (PEGs). They proposed the improved strategy, that is, I-ACO algorithms, which include the designed direction factor, blocking rule, and smoothening rule. Other papers, for example, $[19,20]$, have used ACO techniques to handle various routing issues in WSN but none of them have outperformed while considering cluster based ad hoc sensor networks. In this paper, we have applied the $\mathrm{ABC}$ and $\mathrm{ACO}$ to propose a cluster based routing plan for WSNs.

The fundamental thought in the proposed algorithm (Algorithm 1) is the choice of a $\mathrm{CH}$ and $\mathrm{SCH}$ that can reduce the distance between themselves and their neighbours within a cluster. Each node is installed in such way that it has equal distance from each of its neighbouring nodes and this distance is the same for every node of the network. The square shaped sensing field is divided into subregions and then subregions parts based on optimal clustering mechanism. Finally, among the divided regions, the $\mathrm{CH}$ is selected using the $\mathrm{ABC}$ algorithm and then $\mathrm{CHs}$ use $\mathrm{ACO}$ algorithm, which is an organically propelled standard for improved methodology, to discover a best route to the BS.

\section{Radio Energy Model}

Our energy model for the WSN is taking into consideration the first-order radio model as reported in [9]. In this firstorder radio model, a radio energy disseminated model is proposed in which transmitter chunks the energy while operating the radio electronics and power amplifier; on the other hand recipient disseminates the energy while initiating the radio electronics. The first-order model uses both free space and multipath fading models considering the distance between the sender and the receiver. If the considered distance between the transmitter and receiver is less than $d_{0}$, then the free space model is implemented; else multipath fading will be applicable.

With a specific end goal to send l-bit message over the network both the transmitting and receiving energies will be calculated by the following equations, respectively:

$$
\begin{aligned}
E_{\text {Trans }}(l, d) & =E_{\text {Trans-elec }}(l)+E_{\text {Trans-amp }}(l, d) \\
& = \begin{cases}l E_{\text {elec }}+l E_{\mathrm{fs}} d^{2}, & d \leq d_{0} \\
l E_{\text {elec }}+l E_{\mathrm{mp}} d^{4}, & d>d_{0},\end{cases} \\
E_{\text {Rece }}(l, d) & =E_{\text {Rece-elec }}(l)=l E_{\text {elec }} .
\end{aligned}
$$

The CHs of the cluster expend their energy at three levels, that is, receiving energy to get the signals from their member nodes, aggregation energy to aggregate the signals, and transmitting energy to transmit the signals to other CHs. Additionally, we accept that distance between transmitter and recipient is more prominent than threshold. So energy utilization of $\mathrm{CH}$ for a cycle is given by

$$
\begin{aligned}
E_{\mathrm{CH} / \mathrm{cycle}}= & l E_{\text {Rece-elec }}\left(\frac{N}{k}-1\right)+\frac{N}{k} l E_{\text {DataAgr }} \\
& +l E_{\text {Trans-elec }}+l \epsilon_{\mathrm{amp}} d_{\mathrm{toBS}}^{n} .
\end{aligned}
$$

And member nodes of cluster transmit their acquired signals to their respective $\mathrm{CHs}$. So the energy utilization by the member node is given by

$$
E_{\text {mem-node }}=l E_{\text {elec }}+l \epsilon_{\mathrm{fs}} d_{\text {toCH }}^{n} \text {, }
$$




\section{Assumptions:}

(i) $N$ nodes are uniformly dispersed within a square field.

(ii) Each node has unique ID. Nodes located in the event area are grouped into one cluster.

(iii) Nodes are location-aware and quasi-stationary.

(iv) A sensor can compute the approximate distance based on the received signal strength (RSS), and the radio power can be controlled

(v) A fixed base station can be located inside or outside the network sensor fields.

(vi) All nodes are capable of operating in cluster head mode and sensing mode.

(vii) Data fusion is used to reduce the total data message sent.

Variables:

(i) $D$ is the dimension of the squared region.

(ii) Nodes are the number of nodes in the $(D \times D)$ region.

(iii) $E_{0}$ is the Initial energy of the nodes.

(iv) Alive_Nodes is the number of nodes left after the successful execution of previous round.

(v) $d$ is the distance between the nodes.

(vi) $d_{0}$ is the threshold distance value between the free space and multipath fading model.

(vii) $\epsilon_{\mathrm{mp}}$ is the amplification energy.

(viii) $\epsilon_{\mathrm{fs}}$ is the free space energy consumption.

(ix) $N$ is the set of sensor nodes.

(x) Nodes_C is the nodes in sub-region which is under consideration for $\mathrm{CH}$ selection.

(xi) Energy is the residual energy of the nodes.

(xii) $v_{i j}$ is list of possible nodes positions to become a sub-region $\mathrm{CH}$ computed using $\mathrm{ABC}$ algorithm.

(xiii) $\mathrm{CH}_{-} s$ is the list of $\mathrm{CHs}$ in the round.

//Set up phase

Step 1. Take a region of $D \times D\left(\mathrm{~m}^{2}\right)$ with $N$ number of nodes.

Step 2. Initialize the Nodes $(N)$ with initial Energy $\left(E_{0}\right)$

Step 3. For each round Until Alive_Nodes $>0$, then

Repeat Step 4 to Step 16

function Alive_Nodes (Nodes)

Total_Alive $:=0$

for each node $i:=1$ to length (Nodes) do

if Energy [Nodes $[i]]>0$ then

NODE_STATUS $[i]:=1$

Total_Alive $:=$ Total_Alive +1

Else

NODE_STATUS $[i]:=0$

end if

end for

return Total_Alive \{Returns Alive node count\}

endfunction

Step 4. Compute the distance of each node from other nodes and the BS by using Euclidian distance formula.

$$
\text { dist }=\sqrt{\left(x_{1}-x_{2}\right)^{2}+\left(y_{1}-y_{2}\right)^{2}}
$$

where $x_{1}$ and $x_{2}$ are $x$-coordinates of nodes and $y_{1}$ and $y_{2}$ are $y$-coordinates of the nodes.

Step 5. Compute $K_{\text {opt }}$ for optimal number of sub_regions that can be formed in given region $D \times D\left(\mathrm{~m}^{2}\right)$

and divide the given region $D \times D\left(\mathrm{~m}^{2}\right)$ into $k$ sub_regions based on $K_{\mathrm{opt}}$ value.

function Region Optimum Value $\{$ NODES $\}$

$K_{\mathrm{opt}}:=$ Null

if BS.location is "Outside" then

if $\left(d \leq d_{0}\right)$ then

else

$$
K_{\mathrm{opt}}=\sqrt{\frac{4 N}{5+12 K / D+12 K^{2} / D^{2}}}
$$

$$
\begin{aligned}
& \qquad K_{\mathrm{opt}}=\sqrt{\frac{60 N \epsilon_{\mathrm{fs}}}{\left(7 D^{2}+120 L^{2}+180 L^{4} / D^{2}\right) \epsilon_{\mathrm{mp}}}} \\
& \begin{array}{l}
\text { endif } \\
\text { endif } \\
\text { endurn } K_{\mathrm{opt}}
\end{array}
\end{aligned}
$$


Step 6. For each sub_region repeat Steps 7 and 8.

Step 7. Select $\mathrm{CH}$, for each sub_region using ABC algorithm using function ABC_Cluster_Head\{Energy, Nodes_C\}, Which accepts two arguments, (1) Nodes residual Energy and (2) Nodes in that particular sub_region.

function ABC_Cluster_Head\{Energy, Nodes_C $\}$

Fitness_to_be_CH := Null

for each node $i:=1$ to length(Nodes_C) do

Fitness_to_be_CH := Fitness(Nodes_C $[i] \times$ ID, BS)

end for

Threshold_Energy := NULL

Residual_Energy := NULL

for each node $i:=1$ to length(Nodes_C) do

Residual_Energy $:=$ Residual_Energy +

end for

Energy(Nodes_C $[i]$ )

Threshhold_Energy $=\frac{\text { Residual_Energy }}{\text { length(Nodes_C })}$

for each node $i:=1$ to length(Nodes_C) do

$$
\text { Probability_Nodes_to_CH}[i]=\frac{\text { Fitness_to_be_CH }[i]}{\sum_{j=1}^{\text {length(Nodes_C) }} \text { Fitness to be } \mathrm{CH}[j]}
$$

end for

Threshold_Probability :=1/length(Nodes_C)

// New Position Calculating

for each node $i:=1$ to length(Nodes_C) do

if Probability_Nodes_to_CH[i] >

Threshold_Probability then

rand_ABC := Upper_Bound_ABC +

(Lower_Bound_ABC-Upper_Bound_ABC) $\times$ rand

$v_{i j}:=$ Fitness_Node_CH(Prev_CH_Node $)-$ rand_ABC $\times($ Fitness_Node_CH(Prev_CH_Node $)-$

end if

Fitness_Node_CH (Nodes_C[i]))

end for

Node CH Id := Nodes C_max $\left(v_{i j}\right)$

endfunction

Step 8. For each sub_region, repeat Steps 9 and 10.

Step 9. Divide the sub_region into sub_region_parts based on $K_{\mathrm{opt}}$ value for this sub region.

function Sub_Region_Optimum_Value\{NODES\}

$$
K_{\text {opt }}:=\text { Null }
$$

if $\mathrm{CH}$.location is at "Centre" then

if $\left(d \leq d_{0}\right)$ then

$K_{\mathrm{opt}}=\sqrt{2 N}$

else

$$
K_{\mathrm{opt}}=\sqrt{\frac{60 N \epsilon_{\mathrm{fs}}}{7 D^{2} \epsilon_{\mathrm{mp}}}}
$$

endif

else if $\mathrm{CH}$.location is at "Corner" then

if $\left(d \leq d_{0}\right)$ then

$$
K_{\text {opt }}=\sqrt{\frac{N}{2}}
$$

else

$$
K_{\mathrm{opt}}=\sqrt{\frac{15 N \epsilon_{\mathrm{fs}}}{28 D^{2} \epsilon_{\mathrm{mp}}}}
$$

endif

else if $\mathrm{CH}$.location is at "mid-point of the bottom side" then

if $\left(d \leq d_{0}\right)$ then

$$
K_{\mathrm{opt}}=\sqrt{\frac{4 N}{5}}
$$




$$
\begin{aligned}
& \text { else } \\
& \qquad K_{\mathrm{opt}}=\sqrt{\frac{240 N \epsilon_{\mathrm{fs}}}{193 D^{2} \epsilon_{\mathrm{mp}}}} \\
& \text { endif }
\end{aligned}
$$

end if

Endfunction

Step 10. Select the sub_cluster_head (SCH) for each sub_region_parts based on given fitness formula

(i.e residual energy and distance to sub region $\mathrm{CH}$ ).

[Each SCH is responsible to communicate with $\mathrm{CH}$ of that sub region]

Step 11. Select optimal path for $\mathrm{CH}$ of all sub regions for data transmit to BS using ACO algorithm.

function ACO_Optimal_Path $\left\{\mathrm{CH}_{-}\right.$s, BS\}

for each node $i:=1$ to length $\left(\mathrm{CH}_{-} \mathrm{s}\right)$ do

Fitness $:=$ Fitness $\left(\mathrm{CH}_{-} \mathrm{s}[i], \mathrm{BS}\right)$

end for

for each node $i:=1$ to length(CH_s) do

for each node $j:=1$ to length(CH_s) do

if $i \neq j$ then

$$
\operatorname{Prob}_{k}(p, q)=\frac{[\tau(p, q)]^{\alpha} \cdot[\eta(p, q)]^{\beta}}{\sum_{q \in R_{q}}[\tau(p, q)]^{\alpha} \cdot[\eta(p, q)]^{\beta}}, \quad \text { if } q \notin M^{k}
$$

end if

end for

end for

endfunction

// Steady state phase

Step 12. Nodes wake up and sensed data.

Step 13. Node forwards sensed data to SCH using TDMA with (2).

Step 14. SCH receives sensed data from nodes by using (3) and transmits aggregated data to CH using CDMA with (2).

Step 15. CH aggregates the data receivsed from all SCHs and send to BS using CDMA through a next hop receiver

(i.e $\mathrm{CH}$ of other sub region) with (2).

Step 16. For each region,

if Node_Energy $<E_{\text {th }}$ then go to Step 3.

Algorithm 1: Pseudocode of the proposed algorithm.

where $d_{\text {toCH }}$ is the average length of the member node to their $\mathrm{CH}$.

\section{Squared Optimization Problem}

In [3] the authors show that if the cluster formation is not done in a scalable manner, then the total energy cost of the entire network is maximized at an exponential rate; either the number of clusters is more as compared to the optimal number $\left(K_{\text {opt }}\right)$ of clusters or the number of clusters is less than $K_{\text {opt }}$. If there are a smaller number of clusters, then the considerable number of $\mathrm{CHs}$ has to send their information far, which leads to a higher global energy cost in the system. Furthermore, if there are a bigger number of clusters, then less data aggregation is done locally and for monitoring the entire sensor field, a large number of transmissions are needed. When the uniform distribution of sensor nodes over the sensing field is done, the optimal probability of a sensor node being selected as a $\mathrm{CH}$ as a function of spatial density has been discussed either by simulation $[8,9]$ or analytically $[21,22]$. This is a feasible clustering in the sense that well distribution of energy utilization is done by overall nodes and the total energy utilization is less. This optimal clustering highly relies on the energy model. Similar energy model and analysis are used for the purpose of the study as presented in [14] and determination of the optimal value of being $K_{\text {opt }}$ done analytically. Cluster arrangement algorithm guarantees that the acknowledged number of clusters per cycle is equivalent to $K_{\text {opt }}$. Here, sensor area is supposed to be a square shaped with an area of A. It is required by the optimization problem to calculate the predicted squared distance between member nodes and their $\mathrm{CH}[9,23]$. For extracting a closed-form expression for $K_{\text {opt }}$ accordingly, various special cases are well thought out for obtaining an explicit parametric formula [24].

Suppose the sensing region is a squared shape area $\mathrm{A}$ over which $N$ number of nodes are uniformly deployed. If $K_{\text {opt }}$ clusters are present, then on an average $N / K_{\text {opt }}$ node per cluster, having single $\mathrm{CH}$ and rest cluster member nodes will exist. While receiving signals from the sensors, aggregating signals, and sending aggregated data to the BS there is some dissipation of energy by each and every $\mathrm{CH}$ [9]. Thus the energy dissipated in the $\mathrm{CH}$ during a cycle with the supposition that each and every cycle has one frame is (4) and the energy utilized in a cluster member node is equivalent to (5). The area of each cluster is around $D^{2} / K_{\text {opt }}$. Like in [25] the expected distances are computed from a cluster member 
node to its $\mathrm{CH}$ and from a $\mathrm{CH}$ to the BS. Because of the uniform distribution of $\mathrm{CHs}$ in a $D \times D\left(\mathrm{~m}^{2}\right)$ sensor area, the expected squared region enclosed by each cluster with the $\mathrm{CH}$ positioned at $\left(x_{\mathrm{CH}}, y_{\mathrm{CH}}\right)$ can be computed as given below:

$$
\sqrt{\frac{D^{2}}{K_{\mathrm{opt}}}} \times \sqrt{\frac{D^{2}}{K_{\mathrm{opt}}}},
$$

where $K_{\mathrm{opt}}$ is the optimum number of CHs. The member nodes are also deployed regularly and independently in each cluster, where we have

$$
\begin{aligned}
E\left[x_{\text {mem-nodes }}\right] & =E\left[x_{\mathrm{CH}}\right]=E\left[y_{\text {mem-nodes }}\right] \\
& =E\left[y_{\mathrm{CH}}\right]=\frac{1}{2}\left(\sqrt{\frac{D^{2}}{K_{\mathrm{opt}}}}\right), \\
E\left[\left(x_{\text {mem-nodes }}\right)^{2}\right] & =E\left[\left(x_{\mathrm{CH}}\right)^{2}\right]=E\left[\left(y_{\text {mem-nodes }}\right)^{2}\right] \\
& =E\left[\left(y_{\mathrm{CH}}\right)^{2}\right]=\frac{D^{2}}{3 K_{\mathrm{opt}}} .
\end{aligned}
$$

In this manner the predicted squared length from member nodes to their $\mathrm{CH}$ within a cluster can be computed as

$$
\begin{aligned}
E\left[d_{\mathrm{toCH}}^{2}\right]= & E\left[\left(x_{\text {mem-nodes }}-x_{\mathrm{CH}}\right)^{2}\right] \\
& +E\left[\left(y_{\text {mem-nodes }}-y_{\mathrm{CH}}\right)^{2}\right] \\
= & E\left[\left(x_{\text {mem-nodes }}\right)^{2}\right] \\
& -E\left[x_{\text {mem-nodes }}\right] E\left[x_{\mathrm{CH}}\right]+E\left[\left(x_{\mathrm{CH}}\right)^{2}\right] \\
& +E\left[\left(y_{\text {mem-nodes }}\right)^{2}\right] \\
& -E\left[y_{\text {mem-nodes }}\right] E\left[y_{\mathrm{CH}}\right]+E\left[\left(y_{\mathrm{CH}}\right)^{2}\right] \\
= & \frac{D^{2}}{3 K_{\mathrm{opt}}} .
\end{aligned}
$$

By substituting the value of $d_{\mathrm{toCH}}^{2}$ from (8) in (5), the energy used by each member node per cycle is given by

$$
E_{\text {mem-nodes }}=l E_{\text {Trans-elec }}+\frac{l \epsilon_{\mathrm{fs}} D^{2}}{3 K_{\mathrm{opt}}} .
$$

The energy utilization in the whole cluster during a single cycle can be communicated by using

$$
E_{\text {cluster }}=E_{\mathrm{CH} / \text { cycle }}+\left(\frac{N}{K_{\mathrm{opt}}}-1\right) E_{\text {mem-nodes }} .
$$

Hence $E_{\text {cycle }}$ can be computed as

$$
\begin{aligned}
& E_{\text {cycle }}=K_{\text {opt }} E_{\text {cluster }}=K_{\text {opt }}\left(\left(l E_{\text {Rece-elec }}\left(\frac{N}{K_{\text {opt }}}-1\right)\right.\right. \\
& \left.+l E_{\text {DataAgr }} \frac{N}{K_{\text {opt }}}+E_{\text {Trans-elec }}+l \epsilon_{\mathrm{amp}} d_{\mathrm{toBS}}^{n}\right) \\
& \left.+\left(\left(\frac{N}{K_{\mathrm{opt}}}-1\right)\left(l E_{\text {Trans-elec }}+\frac{l \epsilon_{\mathrm{fs}} D^{2}}{3 K_{\mathrm{opt}}}\right)\right)\right) \\
& =N l E_{\text {Rece-elec }}-K_{\mathrm{opt}} l E_{\text {Rece-elec }}+N l E_{\mathrm{dataAgr}} \\
& +K_{\mathrm{opt}} l E_{\text {Trans-elec }}+K_{\mathrm{opt}} l \epsilon_{\mathrm{amp}} d_{\mathrm{toBS}}^{n}+N l E_{\text {Trans-elec }} \\
& -K_{\mathrm{opt}} l E_{\text {Trans-elec }}+\frac{N l \epsilon_{\mathrm{fs}} D^{2}}{3 K_{\mathrm{opt}}}-\frac{l \epsilon_{\mathrm{fs}} D^{2}}{3}=N l E_{\text {Rece-elec }} \\
& -K_{\mathrm{opt}} l E_{\text {Rece-elec }}+N l E_{\text {DataAgr }}+K_{\mathrm{opt}} l \epsilon_{\mathrm{amp}} d_{\mathrm{toBS}}^{n} \\
& +N l E_{\text {Trans-elec }}+\frac{N}{l} \epsilon_{\mathrm{fs}} D^{2} 3 K_{\mathrm{opt}}-3 \frac{l}{\epsilon_{\mathrm{fs}}} D^{2} .
\end{aligned}
$$

By selecting the feasible solution to the first derivative of (11), that is, $K_{\mathrm{opt}}$, the total consumed energy per cycle, that is, $E_{\text {cycle }}$, can be minimized. The first and second derivative of $E_{\text {cycle }}$ with respect to $K_{\text {opt }}$ are

$$
\begin{aligned}
\frac{\partial E_{\mathrm{cycle}}}{\partial K_{\mathrm{opt}}} & =l \epsilon_{\mathrm{amp}} d_{\mathrm{toBS}}^{n}-l E_{\text {Rece-elec }}-\frac{N \epsilon_{\mathrm{fs}} D^{2}}{3 K_{\mathrm{opt}}^{2}}, \\
\frac{\partial^{2} E_{\mathrm{cycle}}}{\partial^{2} K_{\mathrm{opt}}} & =\frac{2 N l \epsilon_{\mathrm{fs}} D^{2}}{3 K_{\mathrm{opt}}^{3}}>0 .
\end{aligned}
$$

By setting the first derivative with respect to optimal number $K_{\text {opt }}$ to zero, the optimum number of $\mathrm{CHs}$, that is, $K_{\mathrm{opt}}$, can be calculated as

$$
\begin{aligned}
& 0=l \epsilon_{\mathrm{amp}} d_{\mathrm{toBS}}^{n}-l E_{\text {Rece-elec }}-\frac{N \epsilon_{\mathrm{fs}} D^{2}}{3 K_{\mathrm{opt}}^{2}}, \\
& l \epsilon_{\mathrm{amp}} d_{\mathrm{toBS}}^{n}=l E_{\text {Rece-elec }}+\frac{N l \epsilon_{\mathrm{fs}} D^{2}}{3 K_{\mathrm{opt}}^{2}}, \\
& 3 K_{\mathrm{opt}}^{2} l \epsilon_{\mathrm{amp}} d_{\mathrm{toBS}}^{n}=3 K_{\mathrm{opt}}^{2} l E_{\text {Rece-elec }}+N l \epsilon_{\mathrm{fs}} D^{2}, \\
& 3 K_{\mathrm{opt}}^{2}\left(l \epsilon_{\mathrm{amp}} d_{\mathrm{toBS}}^{n}-l E_{\mathrm{Rece-elec}}\right)=N l \epsilon_{\mathrm{fs}} D^{2}, \\
& K_{\mathrm{opt}}^{2}=\frac{N l \epsilon_{\mathrm{fs}} D^{2}}{3 l\left(\epsilon_{\mathrm{amp}} d_{\mathrm{toBS}}^{n}-E_{\text {Rece-elec }}\right)}, \\
& K_{\mathrm{opt}}=\sqrt{\frac{N l \epsilon_{\mathrm{fs}} D^{2}}{3\left(\epsilon_{\mathrm{amp}} d_{\mathrm{toBS}}^{n}-E_{\mathrm{Rece}-\mathrm{elec}}\right)}} .
\end{aligned}
$$

We have shown that a feasible number of clusters depend upon the following parameters: total number of nodes, say $N$, sensing field dimensions $(D)$, average length between sensor 
TABLE 1: Closed-form expressions for squared shaped sensing field $D \times D$ [24].

\begin{tabular}{|c|c|c|c|c|}
\hline Position of BS & Radio model used & Threshold value $d_{0}=\sqrt{\epsilon_{\mathrm{fs}} / \epsilon_{\mathrm{mp}}}$ & Predicted & value of $d_{\mathrm{toBS}}^{2}, d_{\mathrm{toBS}}^{4}$ \\
\hline \multirow{2}{*}{ Centre } & \multirow{7}{*}{ Free space } & \multirow{7}{*}{$d \leq d_{0}$} & & $D^{2}$ \\
\hline & & & & $\overline{6}$ \\
\hline \multirow{2}{*}{ Corner } & & & & $2 D^{2}$ \\
\hline & & & & 3 \\
\hline \multirow{2}{*}{ Side's mid-point } & & & & $5 D^{2}$ \\
\hline & & & & 12 \\
\hline Outside & & & \multicolumn{2}{|c|}{$\frac{5 D^{2}}{12}+D K+K^{2}$} \\
\hline \multirow{2}{*}{ Centre } & \multirow{8}{*}{ Multipath } & \multirow{8}{*}{$d>d_{0}$} & & $7 D^{4}$ \\
\hline & & & & $\overline{180}$ \\
\hline \multirow{2}{*}{ Corner } & & & & $28 D^{4}$ \\
\hline & & & & $\overline{45}$ \\
\hline \multirow{2}{*}{ Side's mid-point } & & & & $193 D^{4}$ \\
\hline & & & & 720 \\
\hline Outside & & & $7 D^{2}$ & $\frac{2 D^{2} L^{2}}{}+L^{4}$ \\
\hline & & & 180 & 3 \\
\hline
\end{tabular}

nodes and BS $\left(d_{\mathrm{toBS}}^{n}\right)$, receiving total energy consumed by sensor nodes $\left(E_{\text {Rece-elce }}\right)$, and transmitter amplifier energy consumption $\left(\epsilon_{\mathrm{amp}}\right)$. Some of the protocols [26-28] neglect the receiving energy dissipation of sensor nodes in exploring the routes and only consider the energy of the transmitter. So we also suppose that receiving circuitry to be very small. If receiving circuitry is large, it will be in the form of a constant overhead that can predominantly make the clustering schemes questionable [24]. The upper and lower range can be attained by putting the least and biggest values of $d_{\text {toBS }}^{n}$ in (13) and can compute the optimal value of $K_{\text {opt }}$. Also the solution of the second derivation of (11) is positive which represents that the value of $K_{\mathrm{opt}}$ defined in (13) results in the least possible total energy consumption in WSNs with the regular node distribution. Once the optimal number of $\mathrm{CHs}$ is obtained, their locations are found by the $\mathrm{ABC}$ algorithm among uniformly distributed sensors. In [24] the authors have derived the expected value of different powers of communication path between the nodes and the BS throughout the sensing field (i.e., $d_{\mathrm{toBS}}^{2}, d_{\mathrm{toBS}}^{4}$ ) as shown in Table 1. If $d \leq$ $d_{0}$ (i.e., for free space model), the expected value of $d_{\text {toBS }}^{2}$ is computed. Also for $d>d_{0}$ (i.e., for two-ray model), the expected value of $d_{\text {toBS }}^{4}$ is computed. Figures 1-3 show the sample plot of the network for different cases of experiments. We have considered one case with BS position (i.e., outside the sensor field) to divide the region into subregions and three cases with $\mathrm{CH}$ position (i.e., centre, corner, and mid-point of bottom side) to divide the subregions into subregion parts. We have used different values of $d_{\text {toBS }}^{2}, d_{\text {toBS }}^{4}$ from Table 1 and submit these values in (13) to calculate the values of $K_{\text {opt }}$ for all these cases. The different values of $K_{\mathrm{opt}}$ from (14), (15) are used to divide the region into subregions and the values of $K_{\text {opt }}$ from (16)-(21) are used to divide the subregions into subregion parts. The simulation of the network environment is executed and the nature of sensor nodes for every round of experiments is visualized in Figures 1-3. These figures show the location of sensor nodes and BS. The green colour

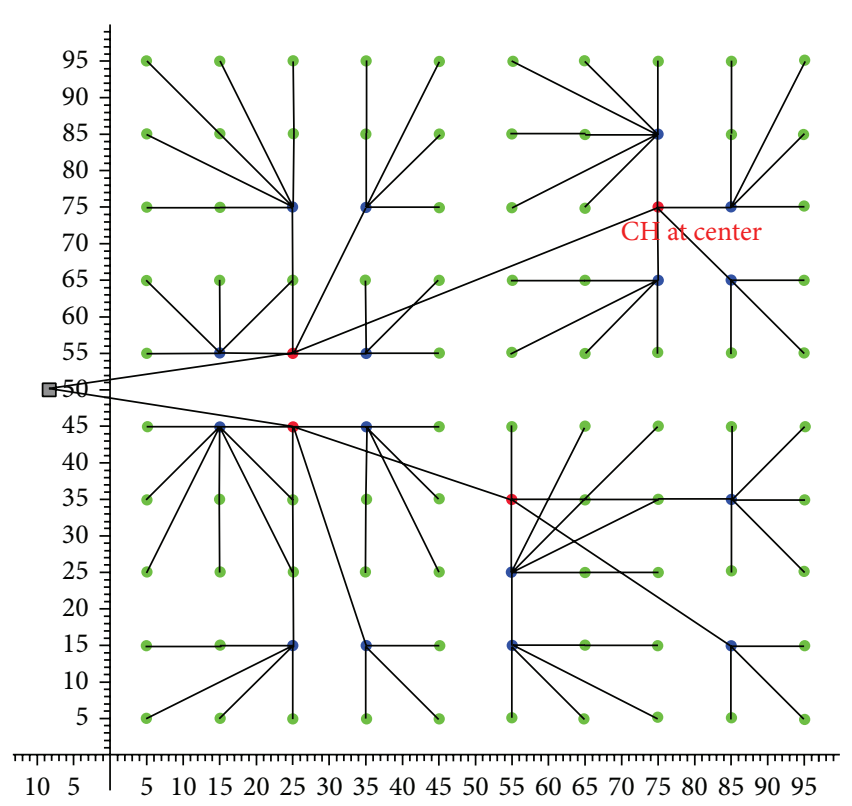

FIgUre 1: Square shaped network field where $\mathrm{CH}$ is located at the centre.

indicates the normal sensor nodes, blue colour indicates the $\mathrm{SCH}$, and red colour indicates the $\mathrm{CH}$. The square box symbol outside the $y$-axis indicates the BS node.

Case 1. BS is placed at outside of the squared network field.

(a) If $d \leq d_{0}$, then $K_{\mathrm{opt}}$ from (13) can be calculated as

$$
\begin{aligned}
& K_{\mathrm{opt}}=\sqrt{\frac{N \epsilon_{\mathrm{fs}} D^{2}}{3\left(\epsilon_{\mathrm{amp}} d_{\mathrm{toBS}}^{2}-E_{\text {Rece-elec }}\right)},} \\
& K_{\mathrm{opt}}=\sqrt{\frac{N \epsilon_{\mathrm{fs}} D^{2}}{3 \epsilon_{\mathrm{fs}}\left(5 D^{2} / 12+D K+K^{2}\right)}},
\end{aligned}
$$




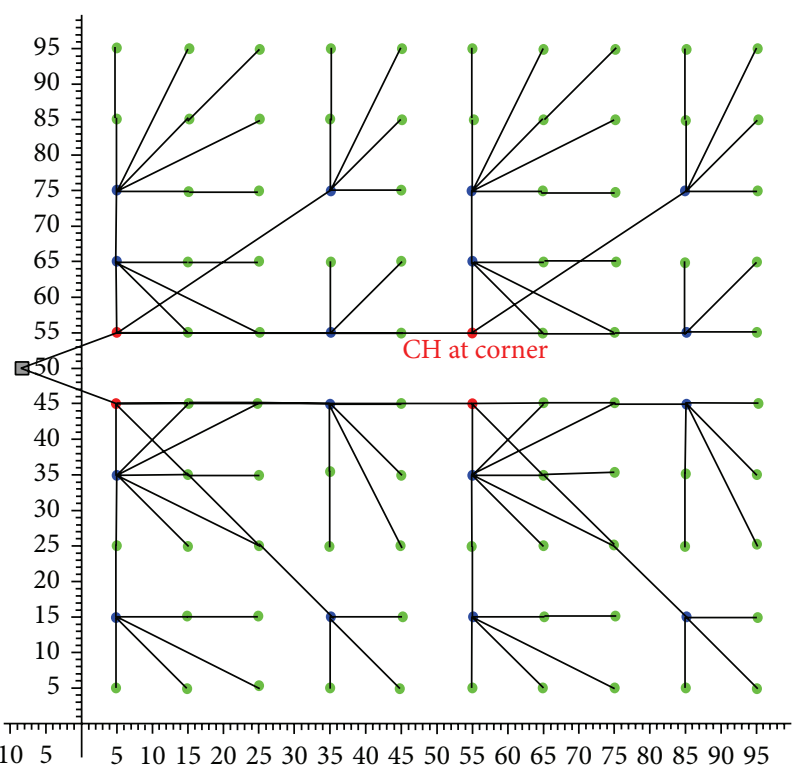

Figure 2: Square shaped network field where $\mathrm{CH}$ is located at the corner.

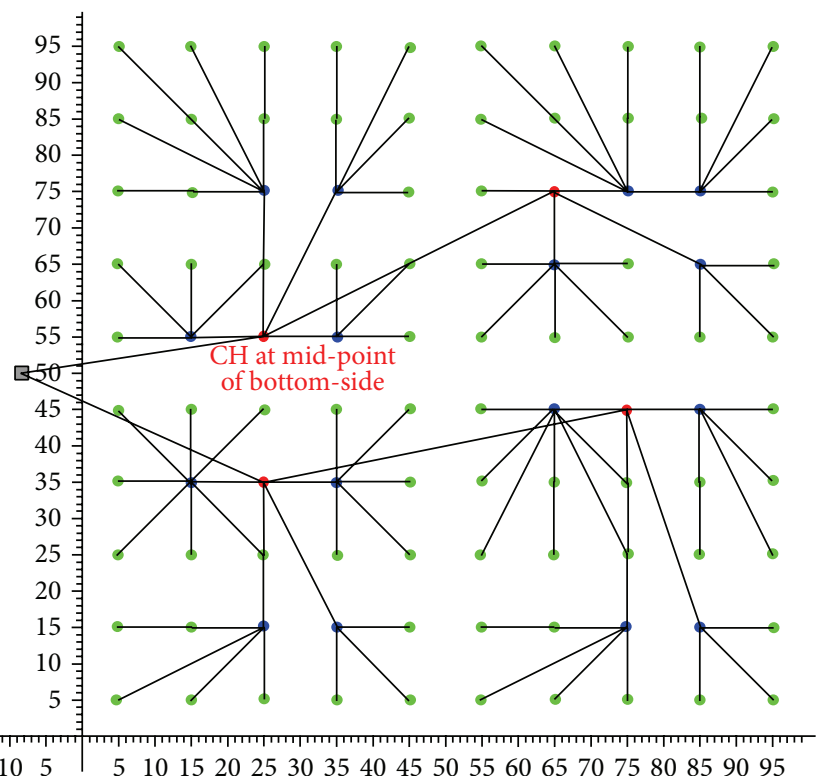

Figure 3: Square shaped network field where $\mathrm{CH}$ is located at the mid-point of bottom side.

$$
K_{\mathrm{opt}}=\sqrt{\frac{4 N}{5+12 K / D+12 K^{2} / D^{2}}} .
$$

(b) If $d>d_{0}$, then $K_{\mathrm{opt}}$ from (13) can be calculated as

$$
K_{\mathrm{opt}}=\sqrt{\frac{N \epsilon_{\mathrm{fs}} D^{2}}{3\left(\epsilon_{\mathrm{amp}} d_{\mathrm{toBS}}^{4}-E_{\text {Rece-elec }}\right)}},
$$

$$
\begin{aligned}
& K_{\mathrm{opt}}=\sqrt{\frac{N \epsilon_{\mathrm{fs}} D^{2}}{3 \epsilon_{\mathrm{mp}}\left(7 D^{4} / 180+2 D^{2} L^{2} / 3+L^{4}\right)}}, \\
& K_{\mathrm{opt}}=\sqrt{\frac{60 N \epsilon_{\mathrm{fs}}}{\left(7 D^{2}+120 L^{2}+180 L^{4} / D^{2}\right) \epsilon_{\mathrm{mp}}}} .
\end{aligned}
$$

Case 2. $\mathrm{CH}$ is located at the centre of the squared network field as shown in Figure 1.

(a) If $d \leq d_{0}$, then $K_{\text {opt }}$ from (13) can be calculated as

$$
\begin{aligned}
& K_{\mathrm{opt}}=\sqrt{\frac{N \epsilon_{\mathrm{fs}} D^{2}}{3\left(\epsilon_{\mathrm{amp}} d_{\mathrm{toBS}}^{2}-E_{\text {Rece-elec }}\right)}}, \\
& K_{\mathrm{opt}}=\sqrt{\frac{N \epsilon_{\mathrm{fs}} D^{2}}{3 \epsilon_{\mathrm{fs}}\left(D^{2} / 6\right)}} \\
& K_{\mathrm{opt}}=\sqrt{2 N}
\end{aligned}
$$

(b) If $d>d_{0}$, then $K_{\text {opt }}$ from (13) can be calculated as

$$
\begin{aligned}
& K_{\mathrm{opt}}=\sqrt{\frac{N \epsilon_{\mathrm{fs}} D^{2}}{3\left(\epsilon_{\mathrm{amp}} d_{\mathrm{toBS}}^{4}-E_{\text {Rece-elec }}\right)}}, \\
& K_{\mathrm{opt}}=\sqrt{\frac{N \epsilon_{\mathrm{fs}} D^{2}}{3 \epsilon_{\mathrm{mp}}\left(7 D^{4} / 180\right)}}, \\
& K_{\mathrm{opt}}=\sqrt{\frac{60 N \epsilon_{\mathrm{fs}}}{7 D^{2} \epsilon_{\mathrm{mp}}}} .
\end{aligned}
$$

Case 3. $\mathrm{CH}$ is located at the corner of the squared network field as shown in Figure 2.

(a) If $d \leq d_{0}, K_{\text {opt }}$ from (13) can be calculated as

$$
\begin{aligned}
& K_{\mathrm{opt}}=\sqrt{\frac{N \epsilon_{\mathrm{fs}} D^{2}}{3\left(\epsilon_{\mathrm{amp}} d_{\mathrm{toBS}}^{2}-E_{\text {Rece-elec }}\right)}}, \\
& K_{\mathrm{opt}}=\sqrt{\frac{N \epsilon_{\mathrm{fs}} D^{2}}{3 \epsilon_{\mathrm{fs}}\left(2 D^{2} / 3\right)}}, \\
& K_{\mathrm{opt}}=\sqrt{\frac{N}{2}} .
\end{aligned}
$$


(b) If $d>d_{0}, K_{\mathrm{opt}}$ from (13) can be calculated as

$$
\begin{aligned}
& K_{\mathrm{opt}}=\sqrt{\frac{N \epsilon_{\mathrm{fs}} D^{2}}{3\left(\epsilon_{\mathrm{amp}} d_{\mathrm{toBS}}^{4}-E_{\text {Rece-elec }}\right)},} \\
& K_{\mathrm{opt}}=\sqrt{\frac{N \epsilon_{\mathrm{fs}} D^{2}}{3 \epsilon_{\mathrm{mp}}\left(28 D^{4} / 45\right)},} \\
& K_{\mathrm{opt}}=\sqrt{\frac{15 N \epsilon_{\mathrm{fs}}}{28 D^{2} \epsilon_{\mathrm{mp}}}} .
\end{aligned}
$$

Case 4. $\mathrm{CH}$ is positioned at the mid-point of the bottom side of the squared network field with the origin remaining in the bottom left corner as shown in Figure 3.

(a) If $d \leq d_{0}$, then $K_{\text {opt }}$ from (13) can be calculated as

$$
\begin{aligned}
& K_{\mathrm{opt}}=\sqrt{\frac{N \epsilon_{\mathrm{fs}} D^{2}}{3\left(\epsilon_{\mathrm{amp}} d_{\mathrm{toBS}}^{2}-E_{\text {Rece-elec }}\right)}}, \\
& K_{\mathrm{opt}}=\sqrt{\frac{N \epsilon_{\mathrm{fs}} D^{2}}{3 \epsilon_{\mathrm{fs}}\left(5 D^{2} / 12\right)}}, \\
& K_{\mathrm{opt}}=\sqrt{\frac{4 N}{5}} .
\end{aligned}
$$

(b) If $d>d_{0}$, then $K_{\mathrm{opt}}$ from (13) can be calculated as

$$
\begin{aligned}
& K_{\mathrm{opt}}=\sqrt{\frac{N \epsilon_{\mathrm{fs}} D^{2}}{3\left(\epsilon_{\mathrm{amp}} d_{\mathrm{toBS}}^{4}-E_{\text {Rece-elec }}\right)}}, \\
& K_{\mathrm{opt}}=\sqrt{\frac{N \epsilon_{\mathrm{fs}} D^{2}}{3 \epsilon_{\mathrm{mp}}\left(193 D^{4} / 720\right)}}, \\
& K_{\mathrm{opt}}=\sqrt{\frac{240 N \epsilon_{\mathrm{fs}}}{193 D^{2} \epsilon_{\mathrm{mp}}}} .
\end{aligned}
$$

\section{Protocol Description}

5.1. Artificial Bee Colony Approach. A recent swarm intelligent approach motivated by the gifted metaheuristic behavior of honey bees [6] is known as artificial bee colony algorithm, that is, $\mathrm{ABC}$, which was invented by Karaboga et al. ABC is a swarm intelligence approach which is being attracted by the foregoing nature of real time bees. Bee colony optimization consumes three kinds of bees, namely, employed bees, onlookers, and scout bees. The basic behavior of these bees is as follows.

Scout Bees. These bees randomly move throughout the searching space and try to explore new food resources and calculate their fitness function.

Employed Bees. Employed bees are currently employing and exploring a specific food resource and calculate the new fitness value. They communicate information about this specific food resource with onlooker bees waiting in the beehive by performing waggle dance. This information can be amount of nectar, direction of food resource, and its distance from the beehive.

Onlooker Bees. If the new fitness value is better than the value calculated by the scout bees then select the employed bee and recruit onlookers to pursue the visited routes by employed bees and calculate fitness function. Choose the onlooker bee with best fitness function.

In artificial bee colony, where the exploitation process is performed by an onlooker and employed bees in the searching environment, scout bees manage exploration of food resources.

At the initial stage bee colony algorithm generates a population of these three types of bees along with their food resource locations through a number of iterations starting from $I=0$ up to $I_{\max }$. The food resources are flower petals and the food source positions are called solutions that have different amount of nectar which is known as the fitness function. Each solution $s_{i}$ is a $d$-dimensional solution vector, where $i=1,2,3, \ldots, z, d$ is number of optimization variables, and $z$ denotes number of onlooker or employed bees which is equal to the food source locations. Depending upon the visual information of the food resource (i.e., its shape, colour, and fragrance) the employed bees update the solutions in their memory and evaluate the nectar amount of the newly generated food resource. If the newly discovered food resource is better than the previous one in terms of fitness value, then bees keep the newly generated solutions in their memory and neglect the previous solution or vice versa. At the second stage of iteration, once all the employed bees had discovered food resources, bees communicate statistics regarding the food resources with onlooker bees sitting in beehive by performing a special dance called waggle dance. The food resource statistics can be quantity of nectar, its direction, and distance from the beehive. When onlooker bees get the information about food resources by the employed bees, they choose the favourable food resource. Depending upon the fitness value, the probability $P_{i}$ [29] for an onlooker bee to choose the food resource is evaluated by the given equation:

$$
P_{i}=\frac{\text { fitness }_{i}}{\sum_{n=1}^{z} \text { fitness }_{i}},
$$

where fitness $s_{i}$ is the fitness function of the solution $i$ which is proportional to the nectar quantity of food resource at the position $i$ and $z$ is the number of food sources.

To generate optimal food source location [29], bee colony uses the given equation

$$
x_{i j}^{\prime}=x_{i j}+r_{i j}\left(x_{i j}-x_{k j}\right)
$$

where $j \in\{1,2, \ldots, D\}$ are indexes that are arbitrarily selected and $k \in\{1,2, \ldots, m\}$, where $k$ is determined arbitrarily that 
must be different from $i$, where $r_{i j}$ is a random number that lies within $[-1,1]$. It controls the generation of food sources in the neighbourhood of $x_{i j}$ and also shows the comparison of two food locations using bees. Hence, from (23), it is noticed that if the difference in the $x_{i j}$ and $x_{j k}$ parameters reduces, variation towards the $x_{i j}$ solution also gets reduced. Therefore the moment searching process reaches the optimal solution; number of steps will be decreased accordingly. If the parameter value generated by the searching process is greater than the maximum limit, it will be sustainable and if food source location cannot be improved through a preordained number of iterations, then that food resource will be replaced with another food resource discovered by the scout bees. The predefined number of iterations is an important control parameter of the artificial bee colony, so called a limit for rejection. The food resource whose nectar is neglected by the bees is replaced with a new food resource found by the scouts [29] using

$$
\begin{aligned}
x_{i}^{j}=\mathrm{LB}_{i}^{j}+r(0,1) & \left(\mathrm{UB}_{i}^{j}-\mathrm{LB}_{i}^{j}\right) \\
& \forall j \in(1,2, \ldots, D), \forall i \in(1,2, \ldots, N) .
\end{aligned}
$$

Basically bee colony technique carries out various selection procedures.

(i) Global Selection Procedure. This process is followed by onlookers in order to discover favourable regions with some probability to choose the food resource by using (22).

(ii) Local Selection Procedure. This process is executed by employed bees and onlookers using the visual status of food resources.

(iii) Greedy Selection Procedure. Onlooker and employed bees execute this procedure in which as long as the nectar quantity of the optimal food resource is larger than the current food resource, the bee skips the current solution and retains the optimal solution generated by using (23).

(iv) Random Selection Procedure. This process is performed by scouts as mentioned in (24).

5.2. Ant Colony Optimization Based Routing in WSN. Ant colony optimization is a swarm intelligence approach that is used to solve complex combinatorial problems [30]. The best example for ACO application is AntNet algorithm which was discovered by Di Caro and Dorigo [31]. ACO considers simulated ants as mobile agents that work collectively and communicate with each other via artificial pheromone trails. In ACO based methodology, each and every ant randomly tries to search a way in the search space using forward and backward movements. Ants are originated from a source node at a regular time interval and while travelling through its neighbouring nodes, ant reaches its last destination so called sink node, say, $d$. At whatever node the ant is, the information about a node has to be interchanged with the destination (i.e., $\mathrm{BS})$; as ants are self-propelling in nature, therefore ants are set in motion. At each node $p$, the probability with which an ant chooses the next node $q$ is given by

$$
\begin{aligned}
& \operatorname{Prob}_{k}(p, q) \\
& \quad= \begin{cases}\frac{[\tau(p, q)]^{\alpha} \cdot[\eta(p, q)]^{\beta}}{\sum_{q \in R_{q}}[\tau(p, q)]^{\alpha} \cdot[\eta(p, q)]^{\beta}}, & \text { if } q \notin M^{k} \\
0 & \text { otherwise, }\end{cases}
\end{aligned}
$$

where $\tau(p, q)$ are the pheromone generated by the backward ants and $\eta(p, q)$ is the estimated heuristic function for energy and distance and $R_{q}$ is the recipient nodes. For node $p, M^{k}$ is the list of nodes previously visited by the ants. $\alpha$ and $\beta$ are two control parameters which restrict the amount of pheromones. Pheromone values are deposited along circular arcs such that each arc $(p, q)$ has a trail value $\tau(r, s) \in[0,1]$. Since the destination hop is a stable base station, therefore the last node of the path for every ant journey is the same. The higher the probability $\operatorname{Prob}_{k}(p, q)$ the higher the chances of the node $q$ being selected. The heuristic value of the node $p$ is expressed by the following equation:

$$
\eta(p, q)=\sum_{i=1}^{n} \frac{E_{c}^{i}}{E_{I}^{i}} \times D\left(\mathrm{BS}_{z}, q_{i}\right),
$$

where $E_{c}^{i}$ is the current energy of the $i$ th node. $E_{I}^{i}$ is the initial energy of the $i$ th node. $D\left(\mathrm{BS}_{z}, q_{i}\right)$ is minimum distance of $\mathrm{CH} q_{i}$ from $\mathrm{BS}_{z}$. Henceforth an ant can choose the next node on the basis of the amount of energy and distance level of the neighbouring nodes. This inferred that if a node has a lower energy level, it means it has a lower probability of being selected and vice versa. The moment forward ant reaches its destination node, a backward ant is originated and the memory of the foraging ant is exchanged to the trailing ant and then the forward ant is released. The trailing ant moves in the same way as the forward ant except in backward direction. While moving hop to hop back to the source node, the trailing ant retains an amount of pheromone, $\Delta \tau^{k}$, on every node which is given by the following equation:

$$
\Delta \tau^{k}=w \cdot\left(\frac{1}{L^{k}}\right)
$$

where $L^{k}$ is the route length of the $k$ th ant in terms of number of visited hops and $w$ is the weighting coefficient. These pheromone qualities are retained in the memory for the future reference. The quality of a node is determined by the measurement of pheromones on the way to its neighbouring hops. Therefore, it is observed that the values of the variables $M^{k}$ and $L^{k}$ have been confined in a memory stored for every ant in every node. However, this space is less than equal to a few bytes. This information is placed onto the edge relating to the foraging ant. To control the quantity of pheromones, pheromone evaporation operation is performed in order to avoid the immense amount of pheromone trails and to favour the searching of new paths. The evaporation mechanism is performed by using the following equation:

$$
\tau_{p q}=(1-\rho) \tau_{p q},
$$


where $0<\rho \leq 1$ is the abandonment of pheromone trail and $\rho$ is the coefficient of stigmergy persistence.

5.3. Proposed Hybrid (ABCACO) Algorithm. The proposed algorithm operates iteratively, where each iteration initiates with a setup phase followed by a steady-state phase. After uniform or homogeneous deployment process of the network, each node initially calculates distance to other nodes and from BS using the Euclidian formula. At the starting of each setup phase, all nodes transfer the information regarding their energy and locations (calculated distances) to the base station. Thus, using this information, the base station computes the average energy level of all hops and decides which node will be chosen as $\mathrm{CH}$ such that node must have sufficient energy and least distance to the BS. After that the value of optimal clustering $\left(K_{\text {opt }}\right)$ is calculated. This value is based on the position of BS, meaning that BS is positioned outside or centred of the square shaped sensing field. On the basis of $K_{\text {opt }}$ and distance, all nodes of the region are divided into square shaped clusters known as subregions. Next, BS selects $\mathrm{CH}$ s at each round by using the $\mathrm{ABC}$ algorithm. In this algorithm, the $\mathrm{CHs}$ selection process is achieved using the fitness function given by (29) which is obtained analytically in which the communication energy and distance are the significant factors that are considered:

$$
\text { fitness }_{i}=\sum_{i=1}^{n} \frac{E_{c}^{i}}{E_{I}^{i}} \times D\left(\mathrm{BS}_{z}, k_{i}\right),
$$

where $D\left(\mathrm{BS}_{z}, k\right)$ is the minimum distance of the node $k_{i}$ from $\mathrm{BS}_{z}$. Again $K_{\mathrm{opt}}$ value is calculated and this value is based on the position of $\mathrm{CH}$; that is, $\mathrm{CH}$ is positioned at the centre, corner, and mid-point of the bottom side of the square shaped sensing field. On the basis of this $K_{\text {opt }}$ value and distance, all the nodes of subregions are further divided into subregions parts. Based on a given fitness formula, $\mathrm{SCH}$ of each $\mathrm{CH}$ is selected for each subregion part. In steadystate phase all nodes are responsible for communicating with their $\mathrm{SCH}$ of that subregion part and each $\mathrm{SCH}$ is responsible for communicating with $\mathrm{CH}$ of that subregion. Each $\mathrm{SCH}$ receives data from its member nodes and aggregates the data. After aggregation, $\mathrm{SCH}$ transfers data to the $\mathrm{CH}$ of that subregion. Again the $\mathrm{CH}$ aggregates the data and sends it to the $\mathrm{BS}$ in a multihop fashion (i.e., $\mathrm{CH}$ to $\mathrm{CH}$ ) using the optimal path given by ACO algorithm. In our case we have considered that ants should always move towards the BS. So for this purpose, the number of hops is always less than or equal to $K_{\mathrm{opt}}$. Depending upon the distance, the optimized hop count is calculated for each $\mathrm{CH}$. Next, ants are starting from $\mathrm{CHs}$ to $\mathrm{BS}$ through the next possible hops and return with finding the optimized next hop. From that optimized hop the same process is repeated for the next optimized hop towards BS and so on. In ACO to accomplish a proficient and strong routing process some key characteristics of distinctive sensor networks are considered. Failure in communication nodes is more feasible in WSNs than traditional networks, as nodes are regularly positioned in unattended places and they use a restricted power supply. So the network should not be affected by a node's breakdown and should be in an adaptive structure to sustain the routing process. A better solution for data transmission is achieved by packet switching. In the packet switched network message is broken up into packets of fixed or variable size. Each packet includes a header that contains the source address, destination address, and other control information. The size of a packet depends upon the type of network and protocol used. No resources are allocated for a packet in advance. Resources are allocated on demand on a first-come, first-served basis. The packets are routed over the network hop to hop. At each hop packet is stored briefly before being transmitted according to the information stored in its header. These types of networks are called store and forward networks. Individual packets may follow different routes to reach the destination. In most protocols the transport layer is responsible for rearranging these packets before routing them on to the destination port numbers. So when failure takes place in a trail, the related data packet cannot reach the destination, that is, BS. Acknowledgment signals are used to achieve guaranteed and reliable delivery, So when an acknowledgment for a data packet is absent, the source node, that is, $\mathrm{CH}$, retransmits that packet to an altered path. Therefore, routing becomes more robust by using acknowledgment related data transmission and maintains different routes. Also in this kind of network some routes would be shorter and set aside for minimum energy costs. Communication on these routes should be made at regular intervals to decrease the total energy consumption cost using these paths. That is, to attain lower energy consumption more data packets should be transmitted along shorter transmission routes.

The setup and steady-state phase of the proposed algorithm are given below.

Setup Phase. In this phase, the operation is divided into cycles. In every cycle, all nodes generate and transmit a message to their $\mathrm{SCH}, \mathrm{CH}$, or base station. The BS uses the proposed algorithm to perform the following steps: to (1) divide region into subregions and further subregion parts, (2) select $\mathrm{CH}$ using $\mathrm{ABC}$ algorithm, and (3) compute the shortest multihop path using ACO algorithm. The process of the setup phase is shown in Figure 4.

Steady-State Phase. In this phase, all nodes know their own duty according to the notification message received in the setup phase. Member nodes sense the data and send to their $\mathrm{SCH}$. Each SCH aggregates data received from its member nodes and sends to the $\mathrm{CH}$ of that subregion. Further, each $\mathrm{CH}$ aggregates data received from $\mathrm{SCHs}$ and sends to the next hop $\mathrm{CH}$ node on the shortest path determined in setup phase. When the energy of any node is less than the threshold energy, then that node will not participate in the $\mathrm{CH}$ or $\mathrm{SCH}$ selection. Otherwise the entire system continues with the setup and steady-state phase. The system continues these cycles until every node's energy has been depleted. The process of the steady-state phase is shown in Figure 5.

\section{Simulation Results}

In this section, the simulation results of cluster based WSN clustering using artificial bee colony (WSNCABC) [32] have 


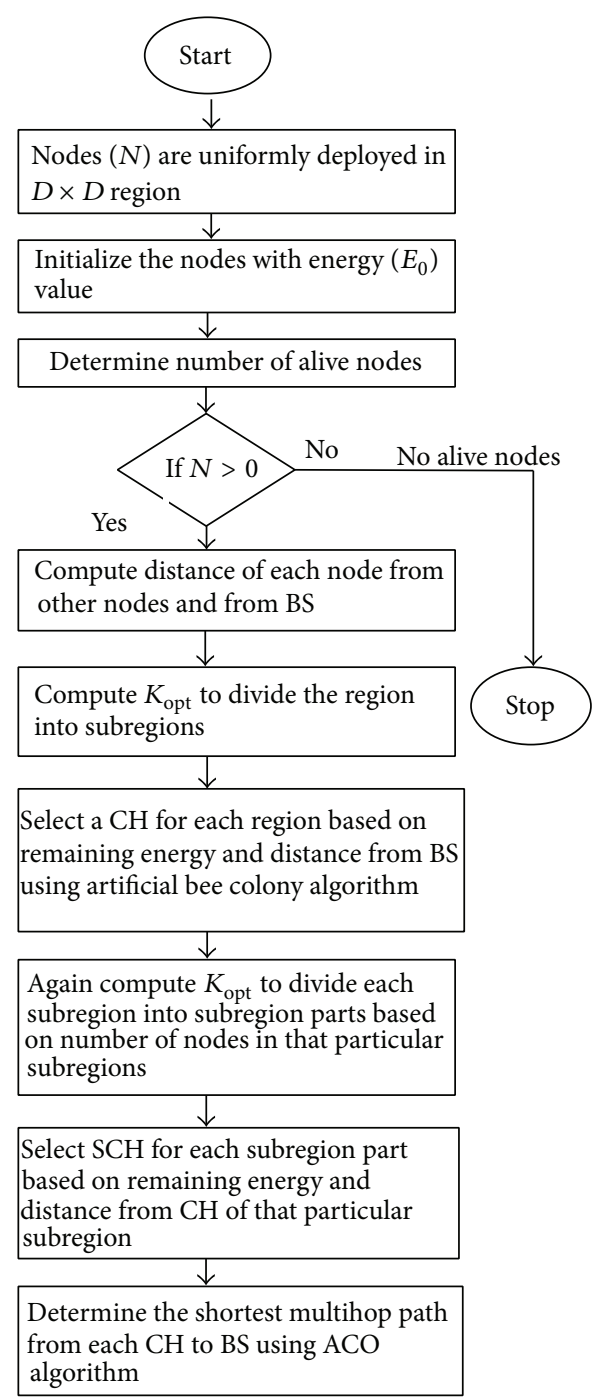

FIGURE 4: Setup phase process.

been compared with a well-known clustering based wireless sensor protocol called "Low Energy Adaptive Clustering Hierarchy" (LEACH) [3]. We used MATLAB 2012b and Oracle JDeveloper $11 \mathrm{~g}$ for evaluation of this approach. The simulations for 100 sensor nodes in a $100 \mathrm{~m} \times 100 \mathrm{~m}$ sensing region and for 225 sensor nodes in a $200 \mathrm{~m} \times 200 \mathrm{~m}$ and $300 \mathrm{~m} \times 300 \mathrm{~m}$ region with equivalent initial energy of $0.5 \mathrm{~J}$ and $1.0 \mathrm{~J}$ have been run. Assume every node has a potential of interacting between base station and the sensor nodes. In the simulations, LEACH and WSNCABC algorithm settings and assumptions are for consistent comparisons. In experiments, a parallel model for MATLAB is used in providing periodical data transfers. Free space and multipath radio propagation models are used in simulations. For comparison, LEACH and WSNCABC were used to verify the success of this approach. A packet level simulation has been performed and utilizes the parameter values of the same physical layer as described in [9]. To get the average results, random topologies had been considered through the simulations. BS is located outside of monitored region. We consider various important aspects as the number of alive nodes per round, remaining energy, goodput (i.e., total number of packets received at the BS), and the stability period of the entire network. Simulation is carried out up to 10 percent of the total number of alive nodes. The simulation parameters are mentioned in Table 2.

After each round the number of live sensors goes down. The network remains alive till the last node. Figures 6-11 depict that the proposed approach has a large number of alive nodes compared against LEACH and WSNCABC. A uniform installation of clusters results in prolonged life of network. While in another two approaches like WSNCABC and LEACH the clusters are installed randomly which may cause nodes to cover a longer distance and may cause poor performance.

Goodput. Goodput is the number of packets delivered successfully per unit time [33].

Figure 12 shows the messages are transmitted more frequently as compared to WSNCABC and LEACH when BS is located at $(-10,50)$ position. The proposed approach depicts 


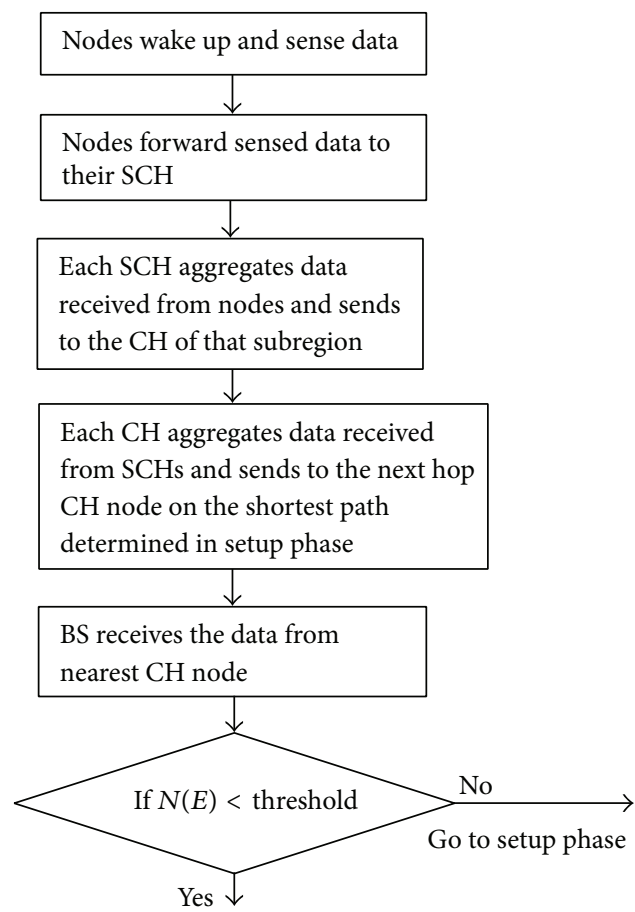

Node will not participate in $\mathrm{CH}$ and $\mathrm{SCH}$ selection

FIGURE 5: Steady-state phase process.

TABLE 2: Simulation parameters.

\begin{tabular}{lc}
\hline Network parameter & Value \\
\hline Field span & Square: $100 \times 100,200 \times 200,300 \times 300$ \\
Numbers of sensor nodes & 100,225 \\
Location of BS & $(-10,50)$ \\
$E_{\text {elec }}$ & $50 \mathrm{~nJ} / \mathrm{bit}$ \\
$E_{\mathrm{fs}}$ & $10 \mathrm{~nJ} / \mathrm{bit} / \mathrm{m}^{2}$ \\
$E_{\mathrm{mp}}$ & $0.0013 \mathrm{pJ} / \mathrm{bit} / \mathrm{m}^{4}$ \\
$E_{\mathrm{DA}}$ & $50 \mathrm{~nJ} / \mathrm{bit} / \mathrm{signal}$ \\
Initial energy & $0.5 \mathrm{~J}, 1.0 \mathrm{~J}$ \\
Channel type & Channel $/$ wireless channel \\
Radio propagation model & Free space, multipath \\
Energy model & Battery \\
Radio bit rate & $1 \mathrm{mbps}$ \\
\hline
\end{tabular}

better results because the $\mathrm{CH}$ s are chosen very carefully after considering the distance of each node from the BS. The CHs are selected based on a high level of energy at least up to a predefined threshold value and the shortest distance from the BS. The graphs indicate that data delivery rate of our approach is far better against WSNCABC and LEACH by a factor of $35 \%$. The other two approaches do not consider these important parameters during $\mathrm{CHs}$ selection which is the reason why they show poor performance.

Stability Period. Stability period is defined when the first node is dead. The stability is a very important parameter in WSNs because after the death of the first node other nodes die

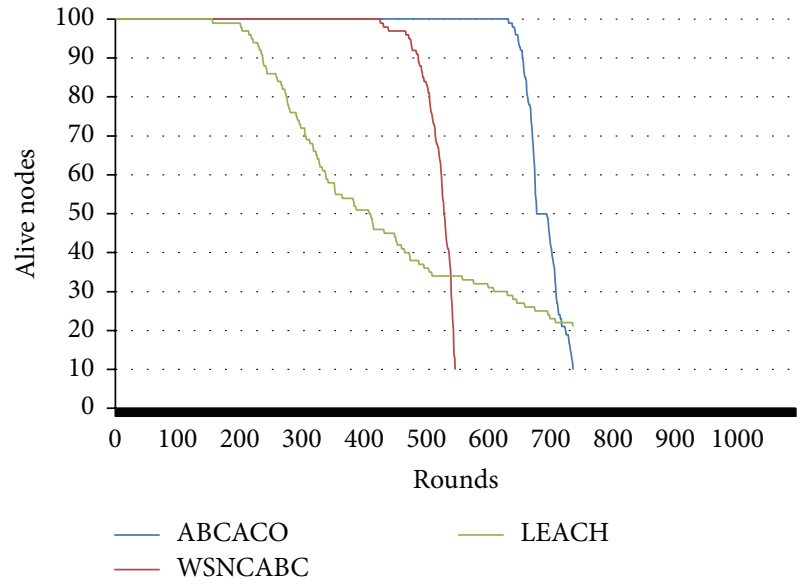

FIGURE 6: Number of alive nodes versus rounds with grid size $100 \times$ 100 and energy $=0.5 \mathrm{~J}$.

gradually which decreases the network lifetime. ABCACO increases the stability period of the network by $48 \%$ against WSNCABC and by $60 \%$ against $\mathrm{LEACH}$, respectively, as shown in Figure 13.

Residual Energy. The residual energy is calculated as follows: residual energy = initial energy - current energy.

Figures 14-19 demonstrate the residual energy of the network versus number of rounds. It clearly indicates that ABCACO algorithm enhances the lifespan of the network over the other two algorithms by consuming less energy. 


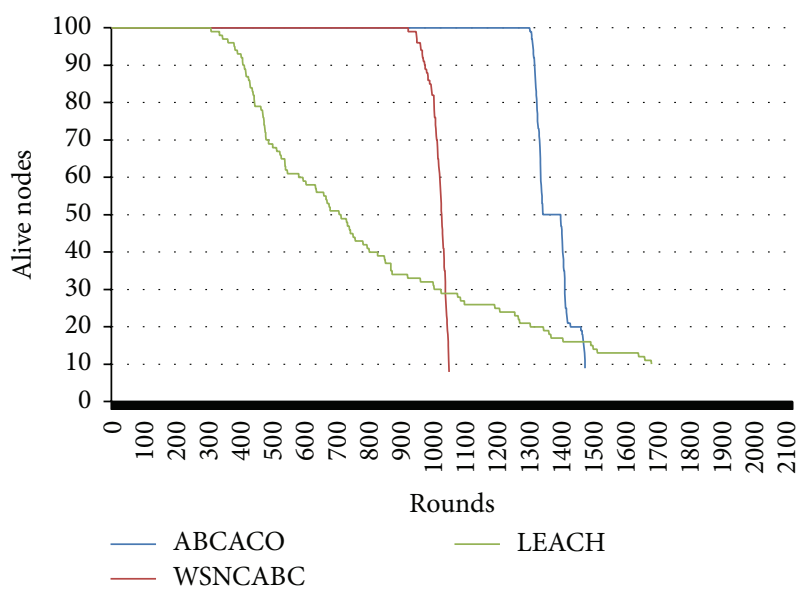

FIGURE 7: Number of alive nodes versus rounds with grid size $100 \times$ 100 and energy $=1 \mathrm{~J}$.

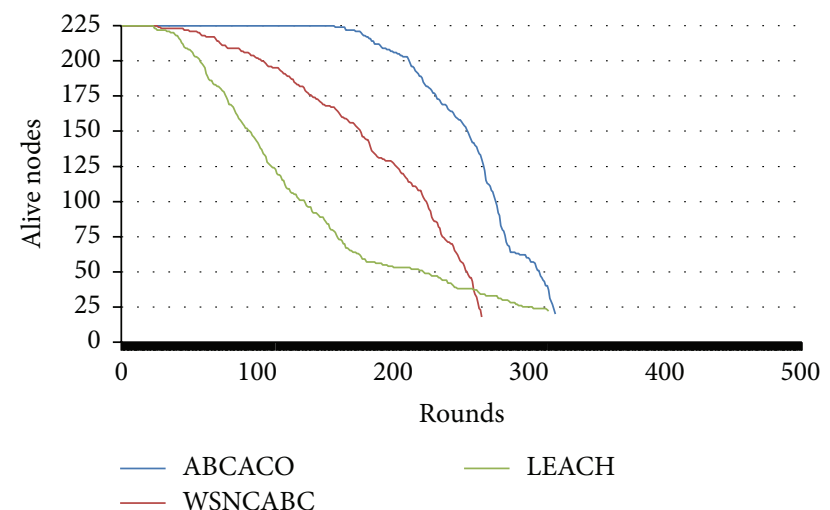

Figure 8: Number of alive nodes versus rounds with grid size $200 \times$ 200 and energy $=0.5 \mathrm{~J}$.

\section{Application}

This paper explains how the wireless sensor network technology helps with fire detection in real time. One of the most appealing features of WSN is environment auditing. Wireless sensor nodes are installed in various parts of the forest which measure temperature, humidity, and biometric data and deliver this collected data to the BS. However, the success of forest fire detection system that is based upon the wireless sensor nodes is restricted due to constrained energy resources of the sensor nodes and the rough environmental settings. Immediate response to fire plays a crucial role in this whole scenario to have the least amount of permanent destruction in the forest. This requires constant surveillance of the area. We decided to study WSN after considering the deficiencies of satellite and camera system. Our proposed architecture not only aims to detect the forest fire effectively and quickly, but also was designed considering the very limitations of sensor nodes.

In our system, sensor nodes work under regular day conditions exempting the period of forest fire such that the wireless sensor nodes will be quite potential under the normal weather conditions and no fire. A distributed (cluster based)

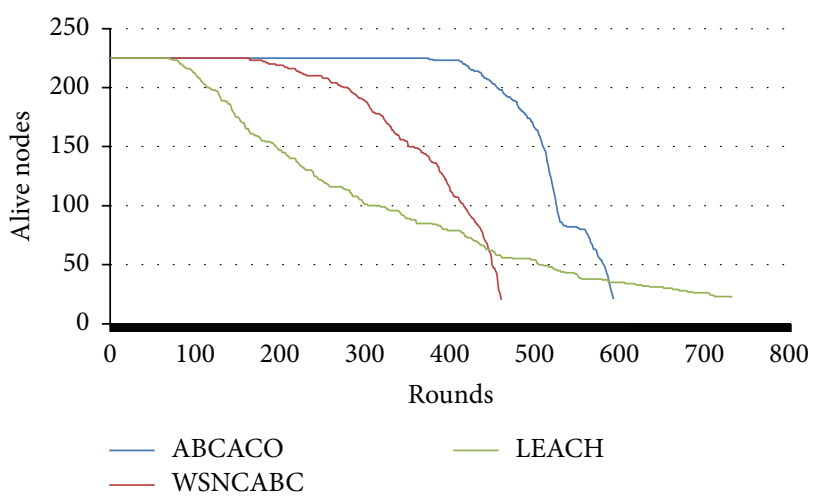

FIgURE 9: Number of alive nodes versus rounds with grid size $200 \times$ 200 and energy $=1 \mathrm{~J}$.

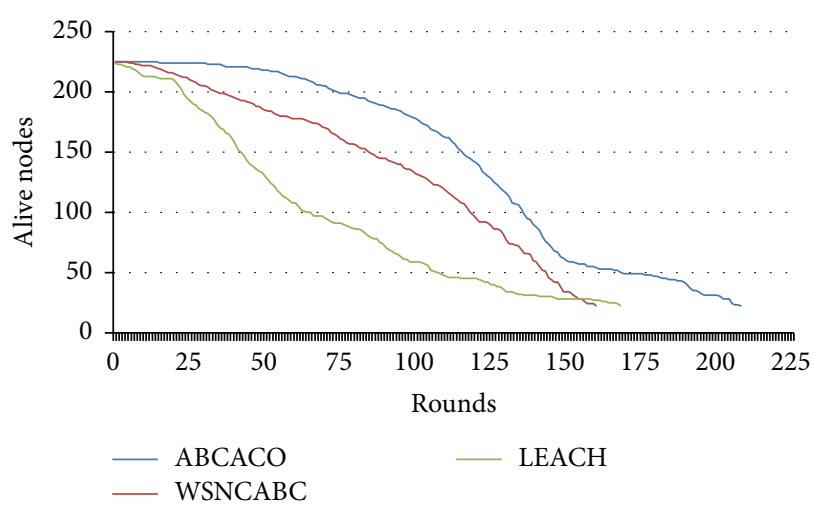

FIGURE 10: Number of alive nodes versus rounds with grid size 300 $\times 300$ and energy $=0.5 \mathrm{~J}$.

approach is used in each sensor node to detect fire threat cautiously in case of abnormally high temperatures to inform the BS about the possibility or occurrence of fire rapidly.

From the literature [34-39], we study that single aspect of environmental monitoring is handled. However, the proposed system takes into account the densely deployed wireless sensor nodes; shortened transmission paths between the sensor nodes enable local computations (i.e., at $\mathrm{SCH}$ and $\mathrm{CH}$ ) and used hybrid swarm intelligent approach with energy and distance parameters for early detection of fire.

Wireless sensor nodes and networks have unique features with many pros and cons of their application in forest fire detection and monitoring. An effective forest fire detection via use of wireless sensor nodes should consider design goals, for example, (a) energy efficiency, (b) earliest forest fire detection, (c) weather resistant structure, and (d) predicting spread and direction of forest fire.

From the abovementioned four design goals of fire detection, we have proposed different strategies: (i) a uniform sensor deployment scheme, (ii) a hierarchical clustered network architecture where $\mathrm{CH}$ is elected by $\mathrm{ABC}$ algorithm with parameters energy and distance from $\mathrm{BS}$ and $\mathrm{SCH}$ is elected by parameters energy and distance from their $\mathrm{CH}$, (iii) an intracluster communication with aggregation of data on $\mathrm{SCH}$, and (iv) intercluster communication (with aggregation of data at $\mathrm{CH}$ ) by using ACO algorithm. 


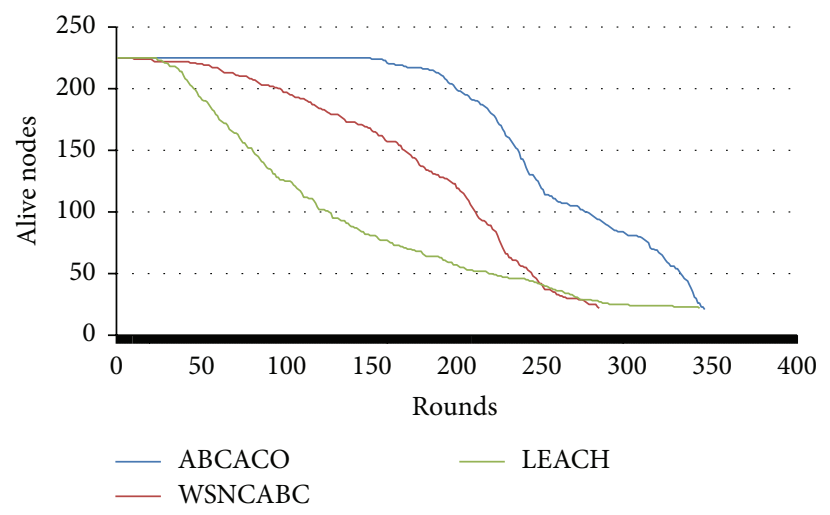

FIGURE 11: Number of alive nodes versus rounds with grid size $300 \times 300$ and energy $=1 \mathrm{~J}$.

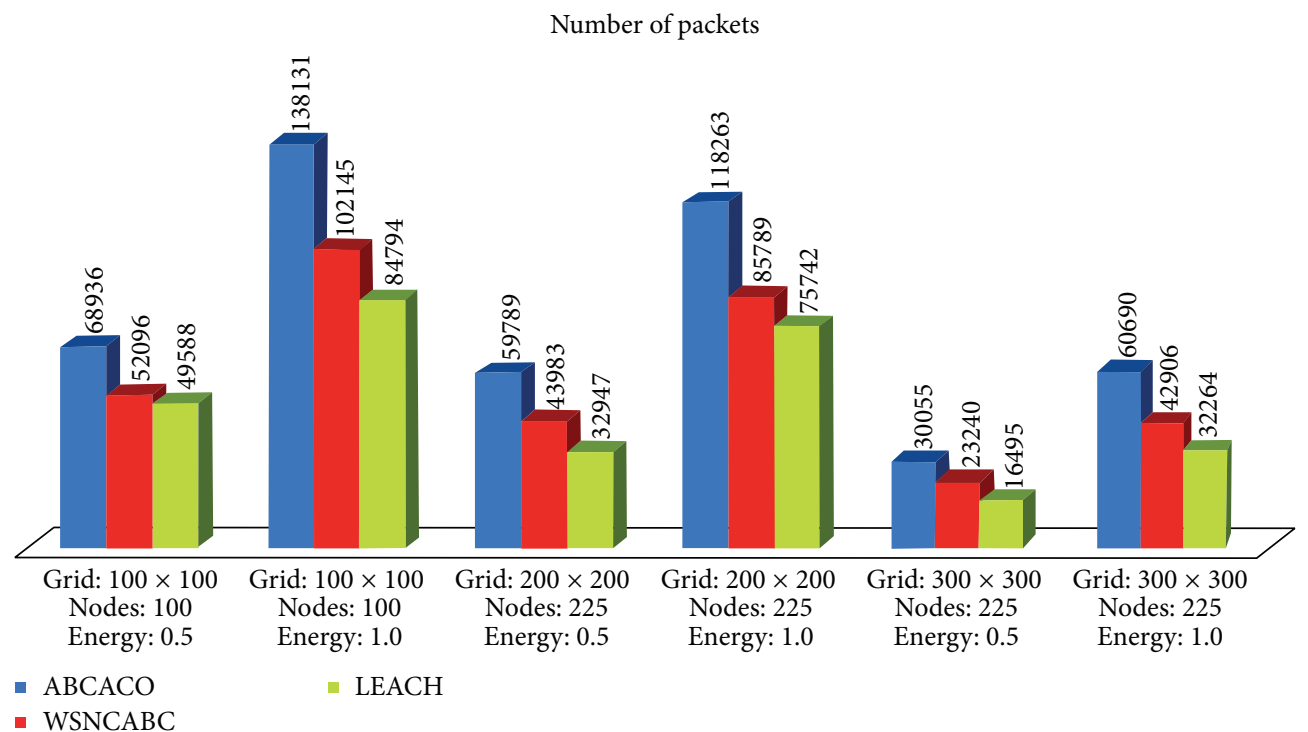

FIGURE 12: Number of successfully received packets at BS located at $(-10,50)$.

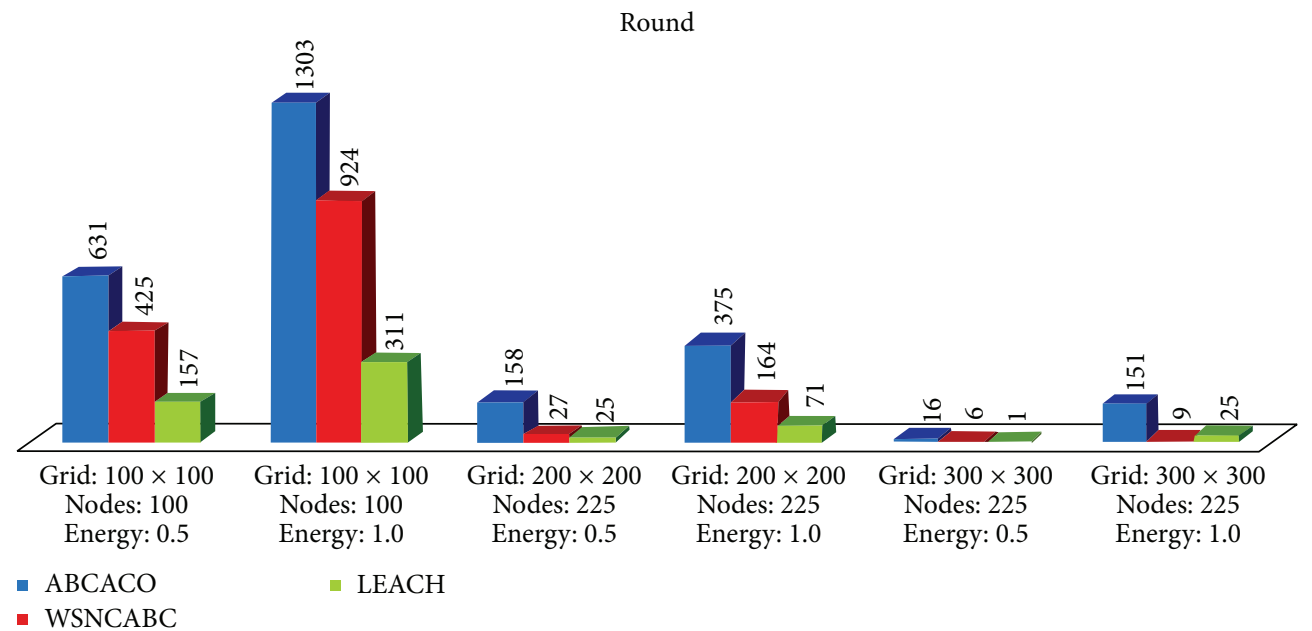

FIGURE 13: Comparison of protocols on the basis of the first node dead. 


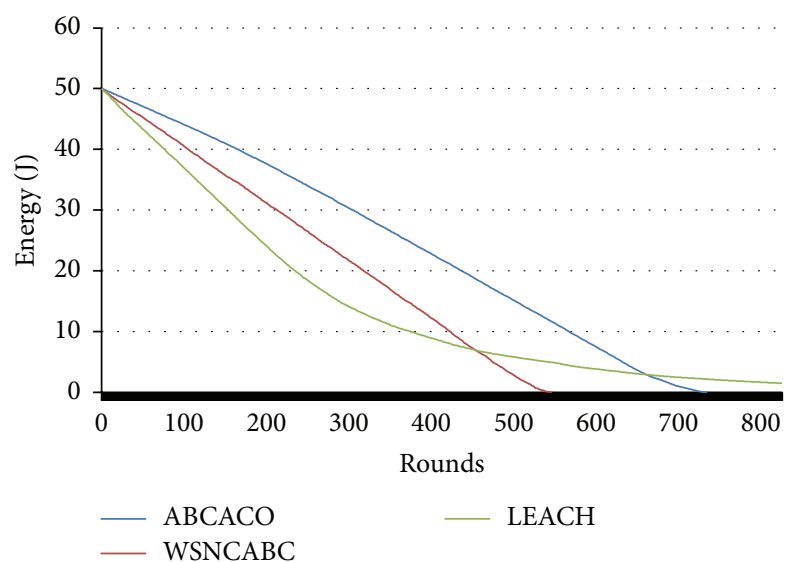

FIGURE 14: Residual energy with grid size $100 \times 100$ and energy $=$ $0.5 \mathrm{~J}$ over rounds.

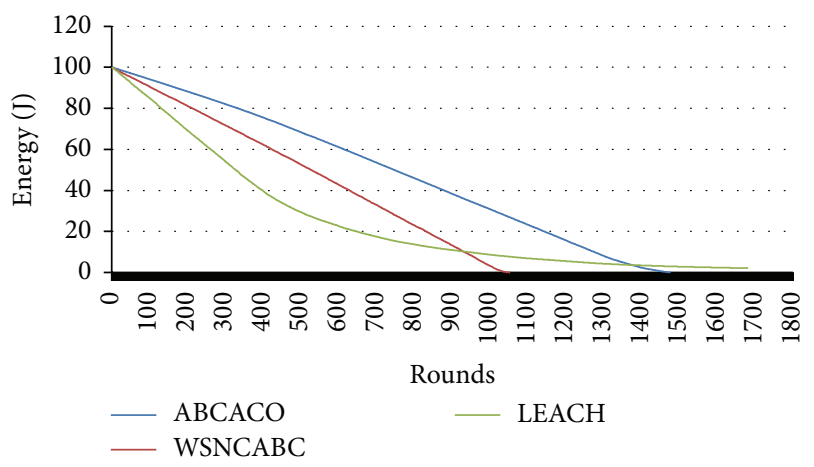

FIGURE 15: Residual energy with grid size $100 \times 100$ and energy $=1 \mathrm{~J}$ over rounds.

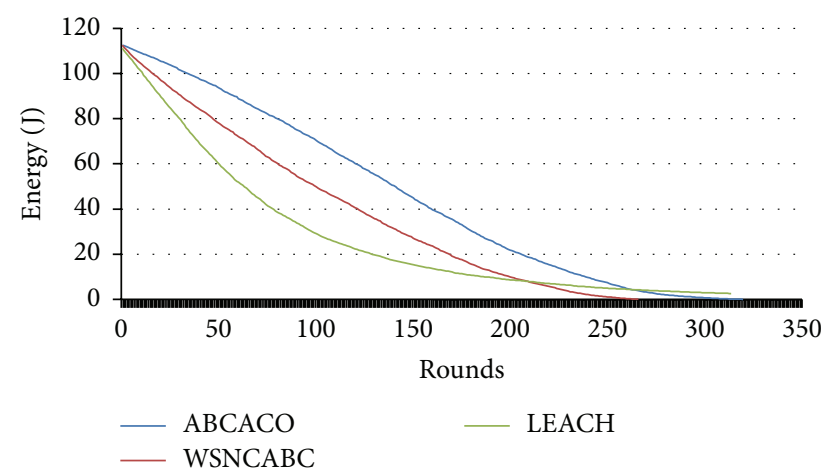

FIGURE 16: Residual energy with grid size $200 \times 200$ and energy $=$ $0.5 \mathrm{~J}$ over rounds.

A sample illustration of a forest fire detection and the communication between the nodes is shown in Figure 20.

\section{Conclusion and Future Scope}

In this paper, we use $\mathrm{ABC}$ because of its simple usage and quick convergence. Additionally, we implement an ACO technique to solve the routing problem that exists in WSNs. We implement both periodical $(\mathrm{ABC})$ and event based

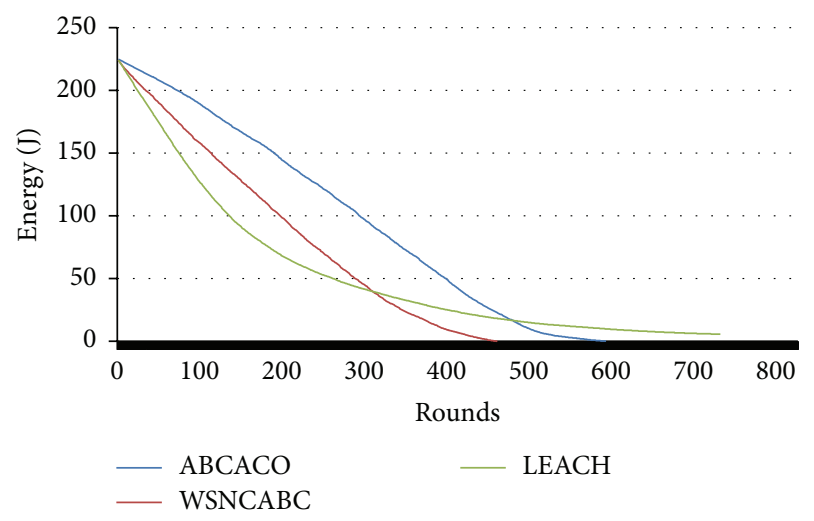

FIGURE 17: Residual energy with grid size $200 \times 200$ and energy $=1 \mathrm{~J}$ over rounds.

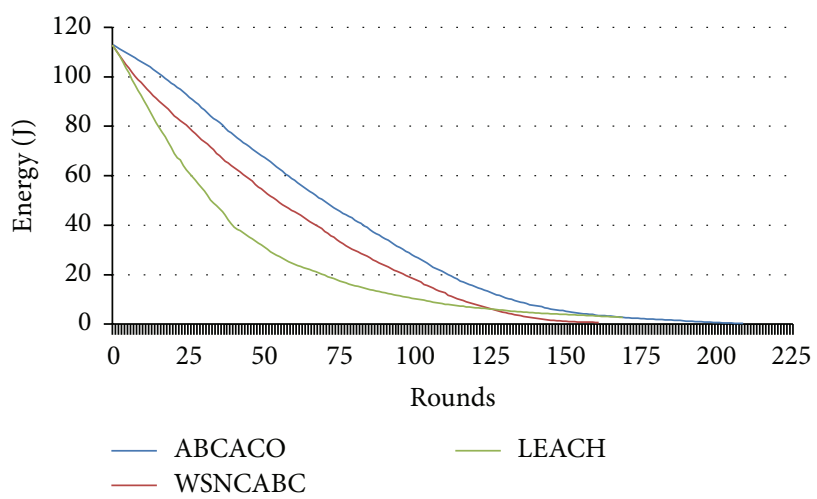

FIGURE 18: Residual energy with grid size $300 \times 300$ and energy $=$ $0.5 \mathrm{~J}$ over rounds.

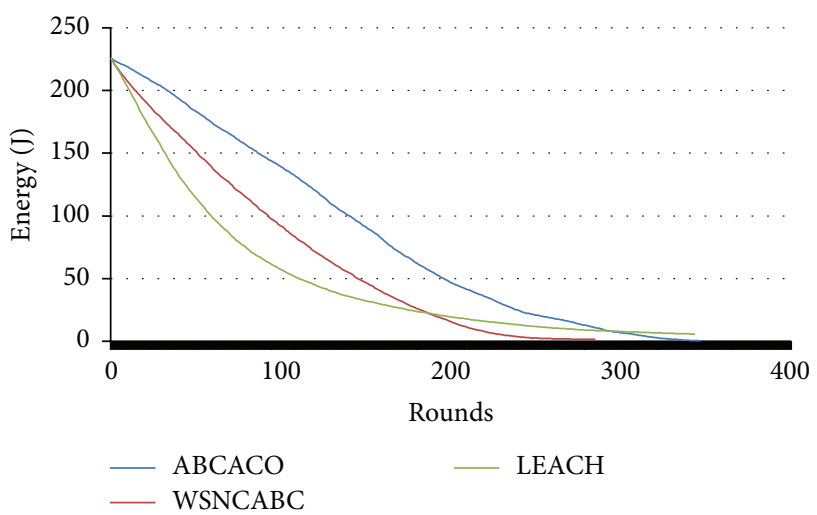

FIGURE 19: Residual energy with grid size $300 \times 300$ and energy $=1 \mathrm{~J}$ over rounds.

(ACO) approaches to develop a new hybrid swarm intelligent energy efficient routing algorithm called ABCACO. In $\mathrm{ABCACO}$, the entire sensing field is divided into an optimal number of subregions and subregion parts based on $K_{\text {opt }}$. The proposed methodology provides a better clustering approach by selecting the CHs uniformly throughout the network area. Since we use hierarchical structure, at the first level, we implement $\mathrm{ABC}$ approach for the selection 


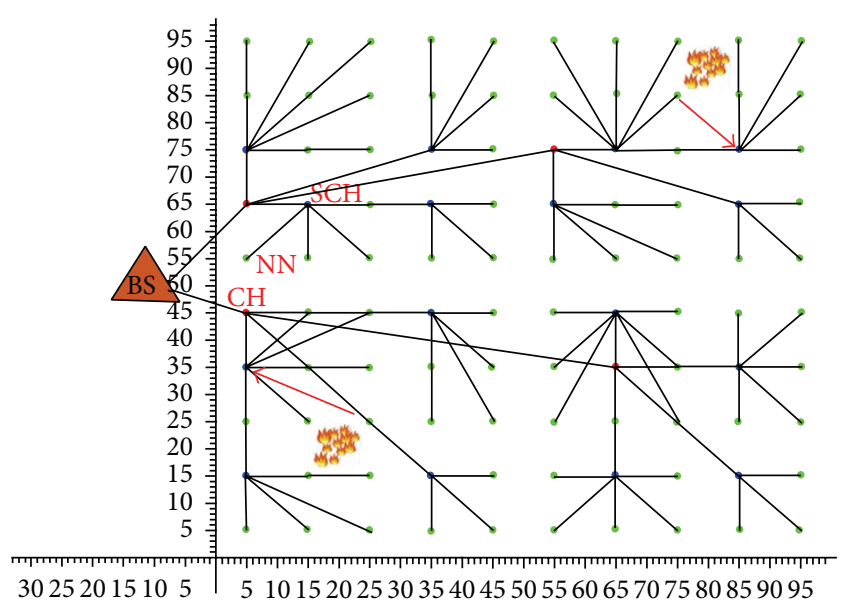

FIgURE 20: Detection of fire event and message forwarding among the nodes.

of $\mathrm{CHs}$ and on the second level a better routing path is evaluated for data transmission by using ACO approach. ABCACO decreases the communication distance by placing the $\mathrm{CH}$ node in the perfect position that provides maximum lifetime of the network. The simulation results show that ABCACO outperforms network performance in terms of lifetime, stability, and goodput as compared to existing algorithms. This paper leads to one step ahead to interdisciplinary research within the biological systems to come up with better bioinspired solutions for real world WSN such as neural swarm system, swarm fuzzy system, and neural fuzzy system. The bioinspired hybrid algorithms open new perspective in the optimization solutions of WSNs.

\section{Conflict of Interests}

The authors declare that there is no conflict of interests regarding the publication of this paper.

\section{References}

[1] I. F. Akyildiz, W. Su, Y. Sankarasubramaniam, and E. Cayirci, "Wireless sensor networks: a survey," Computer Networks, vol. 38, no. 4, pp. 393-422, 2002.

[2] D. Caputo, F. Grimaccia, M. Mussetta, and R. E. Zich, "An enhanced GSO technique for wireless sensor networks optimization," in Proceedings of the IEEE Congress on Evolutionary Computation (CEC '08), pp. 4074-4079, Hong Kong, China, June 2008.

[3] W. R. Heinzelman, A. Chandrakasan, and H. Balakrishnan, "Energy-efficient communication protocol for wireless microsensor networks," in Proceedings of the 33rd Annual Hawaii International Conference on System Sciences, p. 10, IEEE, January 2000.

[4] S. Okdem and D. Karaboga, "Routing in wireless sensor networks using an Ant Colony optimization (ACO) router chip," Sensors, vol. 9, no. 2, pp. 909-921, 2009.

[5] L. Qing, T. Zhi, Y. Yuejun, and L. Yue, "Monitoring in industrial systems using wireless sensor network with dynamic power management," IEEE Sensors Journal, vol. 9, pp. 1596-1604, 2009.
[6] D. Karaboga, S. Okdem, and C. Ozturk, "Cluster based wireless sensor network routing using artificial bee colony algorithm," Wireless Networks, vol. 18, no. 7, pp. 847-860, 2012.

[7] D. Kumar, T. C. Aseri, and R. B. Patel, "Distributed cluster head election (DCHE) scheme for improving lifetime of heterogeneous sensor networks," Tamkang Journal of Science and Engineering, vol. 13, no. 3, pp. 337-348, 2010.

[8] S. Dhar, Energy aware topology control protocols for wireless sensor networks [Ph.D. thesis], 2005.

[9] W. B. Heinzelman, A. P. Chandrakasan, and H. Balakrishnan, "An application-specific protocol architecture for wireless microsensor networks," IEEE Transactions on Wireless Communications, vol. 1, no. 4, pp. 660-670, 2002.

[10] D. Kumar, T. C. A. Patel, and R. B. Patel, "Prolonging network lifetime and data accumulation in heterogeneous sensor networks," International Arab Journal of Information Technology, vol. 7, no. 3, pp. 302-309, 2010.

[11] D. Kumar, T. C. Aseri, and R. B. Patel, "EEHC: energy efficient heterogeneous clustered scheme for wireless sensor networks," Computer Communications, vol. 32, no. 4, pp. 662-667, 2009.

[12] D. Kumar, T. C. Aseri, and R. B. Patel, "EECDA: energy efficient clustering and data aggregation protocol for heterogeneous wireless sensor networks," International Journal of Computers, Communications \& Control, vol. 6, no. 1, pp. 113-124, 2011.

[13] J. Lloret, M. Garcia, D. Bri, and J. R. Diaz, "A cluster-based architecture to structure the topology of parallel wireless sensor networks," Sensors, vol. 9, no. 12, pp. 10513-10544, 2009.

[14] O. Younis and S. Fahmy, "HEED: a hybrid, energy-efficient, distributed clustering approach for ad hoc sensor networks," IEEE Transactions on Mobile Computing, vol. 3, no. 4, pp. 366379, 2004.

[15] S. Lindsey, C. Raghavendra, and K. M. Sivalingam, "Data gathering algorithms in sensor networks using energy metrics," IEEE Transactions on Parallel and Distributed Systems, vol. 13, no. 9, pp. 924-935, 2002.

[16] T. Camilo, C. Carreto, J. S. Silva, and F. Boavida, "An energyefficient ant-based routing algorithm for wireless sensor networks," in Ant Colony Optimization and Swarm Intelligence: 5th International Workshop, ANTS 2006, Brussels, Belgium, September 4-7, 2006. Proceedings, vol. 4150 of Lecture Notes in Computer Science, pp. 49-59, Springer, Berlin, Germany, 2006.

[17] G. Chen, T.-D. Guo, W.-G. Yang, and T. Zhao, "An improved ant-based routing protocol in Wireless Sensor Networks," in Proceedings of the International Conference on Collaborative Computing: Networking, Applications and Worksharing (CollaborateCom '06), pp. 1-7, IEEE, Atlanta, Ga, USA, November 2006.

[18] R. Du, C. L. Chen, X. B. Zhang, and X. P. Guan, "Path planning and obstacle avoidance for PEGs in WSAN: I-ACO based algorithms and implementation," Ad-Hoc and Sensor Wireless Networks, vol. 16, no. 4, pp. 323-345, 2012.

[19] L. Juan, S. Chen, and Z. Chao, "Ant system based anycast routing in wireless sensor networks," in Proceedings of the International Conference on Wireless Communications, Networking and Mobile Computing (WiCom '07), pp. 2420-2423, Shanghai, China, September 2007.

[20] Y. Liu, H. Zhu, K. Xu, and Y. Jia, "A routing strategy based on ant algorithm for WSN," in Proceedings of the 3rd International Conference on Natural Computation (ICNC '07), pp. 685-689, August 2007.

[21] S. Bandyopadhyay and E. J. Coyle, "An energy efficient hierarchical clustering algorithm for wireless sensor networks," in 
Proceedings of the 22nd Annual Joint Conference of the IEEE Computer and Communications IEEE Societies (INFOCOM '03), vol. 3, pp. 1713-1723, IEEE, April 2003.

[22] S. Bandyopadhyay and E. J. Coyle, "Minimizing communication costs in hierarchically-clustered networks of wireless sensors," Computer Networks, vol. 44, no. 1, pp. 1-16, 2004.

[23] L. Qing, Q. Zhu, and M. Wang, "Design of a distributed energyefficient clustering algorithm for heterogeneous wireless sensor networks," Computer Communications, vol. 29, no. 12, pp. 22302237, 2006.

[24] N. Amini, A. Vahdatpour, W. Xu, M. Gerla, and M. Sarrafzadeh, "Cluster size optimization in sensor networks with decentralized cluster-based protocols," Computer Communications, vol. 35, no. 2, pp. 207-220, 2012.

[25] J. Kim, W. Lee, E. Kim, D.-W. Kim, and H. Kim, "Coveragetime optimized dynamic clustering of networked sensors for pervasive home networking," IEEE Transactions on Consumer Electronics, vol. 53, no. 2, pp. 433-441, 2007.

[26] M. Ettus, "System capacity, latency, and power consumption in multihop-routed SS-CDMA wireless networks," in Proceedings of the IEEE Radio and Wireless Conference (RAWCON '98), pp. 55-58, IEEE, Colorado Springs, Colo, USA, August 1998.

[27] T. H. Meng and V. Rodoplu, "Distributed network protocols for wireless communication," in Proceedings of the IEEE International Symposium on Circuits and Systems (ISCAS '98), vol. 4, pp. 600-603, IEEE, Monterey, Calif, USA, May-June 1998.

[28] T. Shepard, "A channel access scheme for large dense packet radio networks," in Proceedings of the Conference Proceedings on Applications, Technologies, Architectures, and Protocols for Computer Communications (SIGCOMM '96), pp. 219-230, August 1996.

[29] D. Karaboga and B. Akay, "A comparative study of artificial Bee colony algorithm," Applied Mathematics and Computation, vol. 214, no. 1, pp. 108-132, 2009.

[30] M. Dorigo and G. Di Caro, "The ant colony optimization metaheuristic," in New Ideas in Optimization, D. Corne, M. Dorigo, and F. Glover, Eds., pp. 11-32, McGraw-Hill, London, UK, 1999.

[31] G. Di Caro and M. Dorigo, "AntNet: distributed stigmergetic control for communications networks," Journal of Artificial Intelligence Research, vol. 9, pp. 317-365, 1998.

[32] D. Karaboga, S. Okdem, and C. Ozturk, "Cluster based wireless sensor network routings using artificial bee colony algorithm," in Proceedings of the IEEE International Conference on Autonomous and Intelligent Systems (AIS '10), pp. 1-5, IEEE, Póvoa de Varzim, Portugal, June 2010.

[33] C. Liu, H. Jiang, Y. Yang, and G. Xu, "Optimal parameters configuration for TCP goodput improvement in CR networks," in Proceedings of the IEEE Spring Congress on Engineering and Technology (S-CET '12), pp. 1-5, IEEE, Xian, China, May 2012.

[34] L. Yu, N. Wang, and X. Meng, "Real-time forest fire detection with wireless sensor networks," in Proceedings of the International Conference on Wireless Communications, Networking and Mobile Computing (WiMob '05), vol. 2, pp. 1214-1217, IEEE, September 2005.

[35] B.-L. Wenning, D. Pesch, A. Timm-Giel, and C. Görg, "Environmental monitoring aware routing: making environmental sensor networks more robust," Telecommunication Systems, vol. 43, no. 1-2, pp. 3-11, 2010.

[36] D. M. Doolin and N. Sitar, "Wireless sensor nodes for wild fire monitoring," in Symposium on Smart Structures and Materials, Proceedings of SPIE, pp. 477-484, San Diego, Calif, USA, 2006.
[37] C. Hartung, R. Han, C. Seielstad, and S. Holbrook, "FireWxNet: a multi-tiered portable wireless system for monitoring weather conditions in wildland fire environments," in Proceedings of the 4th International Conference on Mobile Systems, Applications and Services (MobiSys '06), pp. 28-41, June 2006.

[38] J. Lloret, M. Garcia, D. Bri, and S. Sendra, "A wireless sensor network deployment for rural and forest fire detection and verification," Sensors, vol. 9, no. 11, pp. 8722-8747, 2009.

[39] B. Son and Y.-S. Her, "A design and implementation of forestfires surveillance system based on wireless sensor networks for South Korea mountains," International Journal of Computer Science and Network Security, vol. 6, no. 9, pp. 124-130, 2005. 


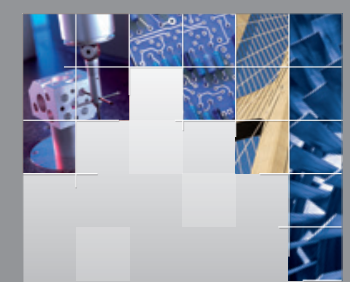

\section{Enfincering}
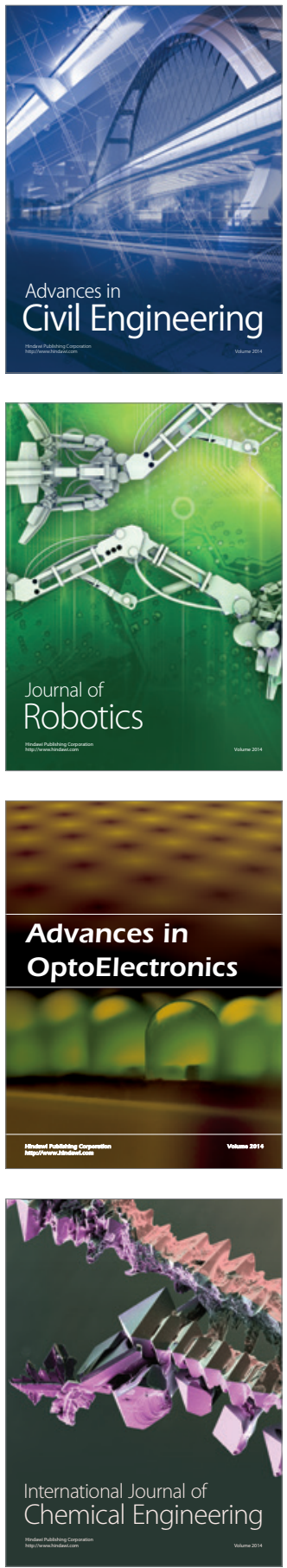

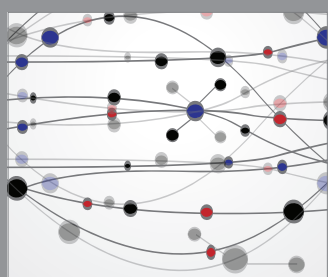

The Scientific World Journal

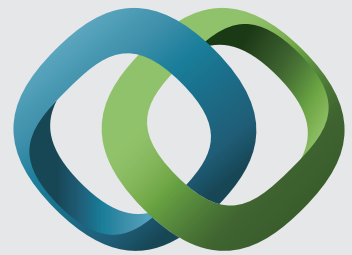

\section{Hindawi}

Submit your manuscripts at

http://www.hindawi.com
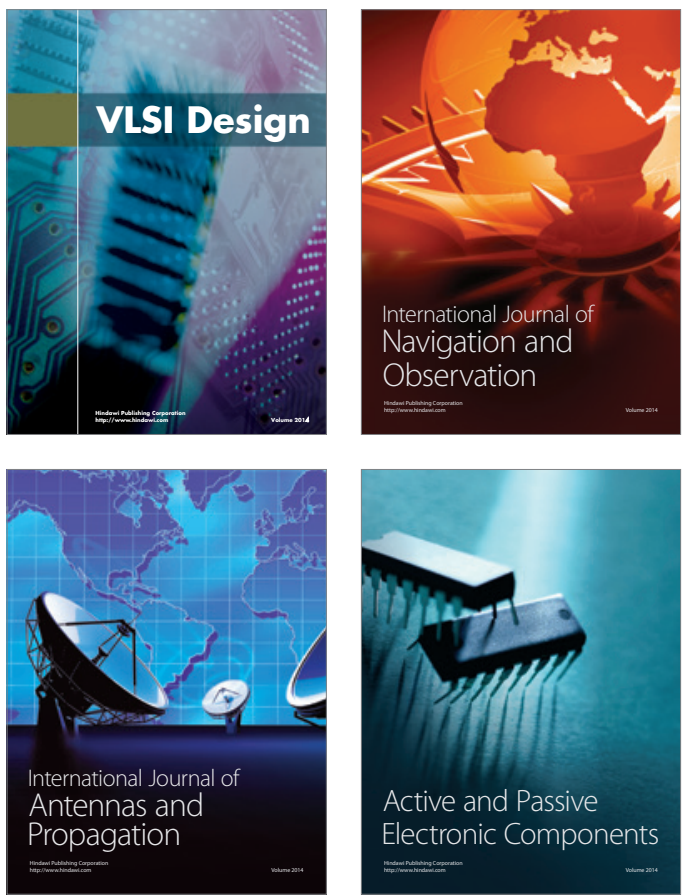
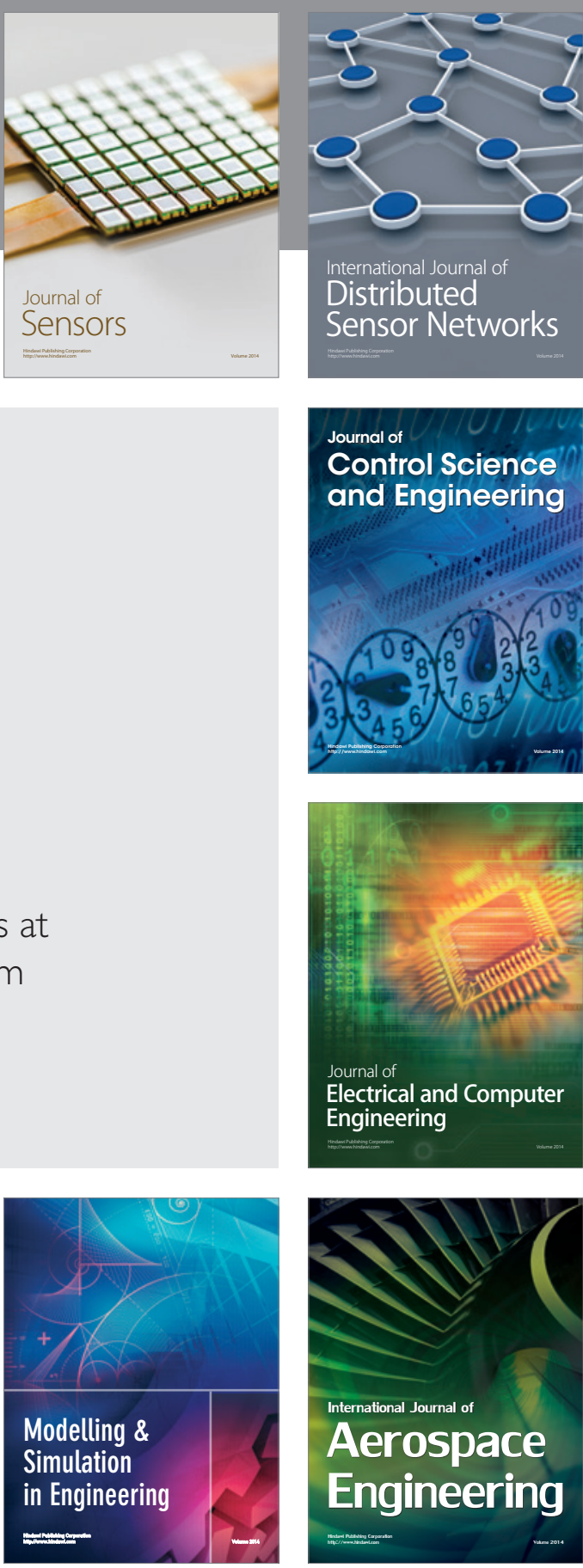

International Journal of

Distributed

Sensor Networks

Journal of

Control Science

and Engineering
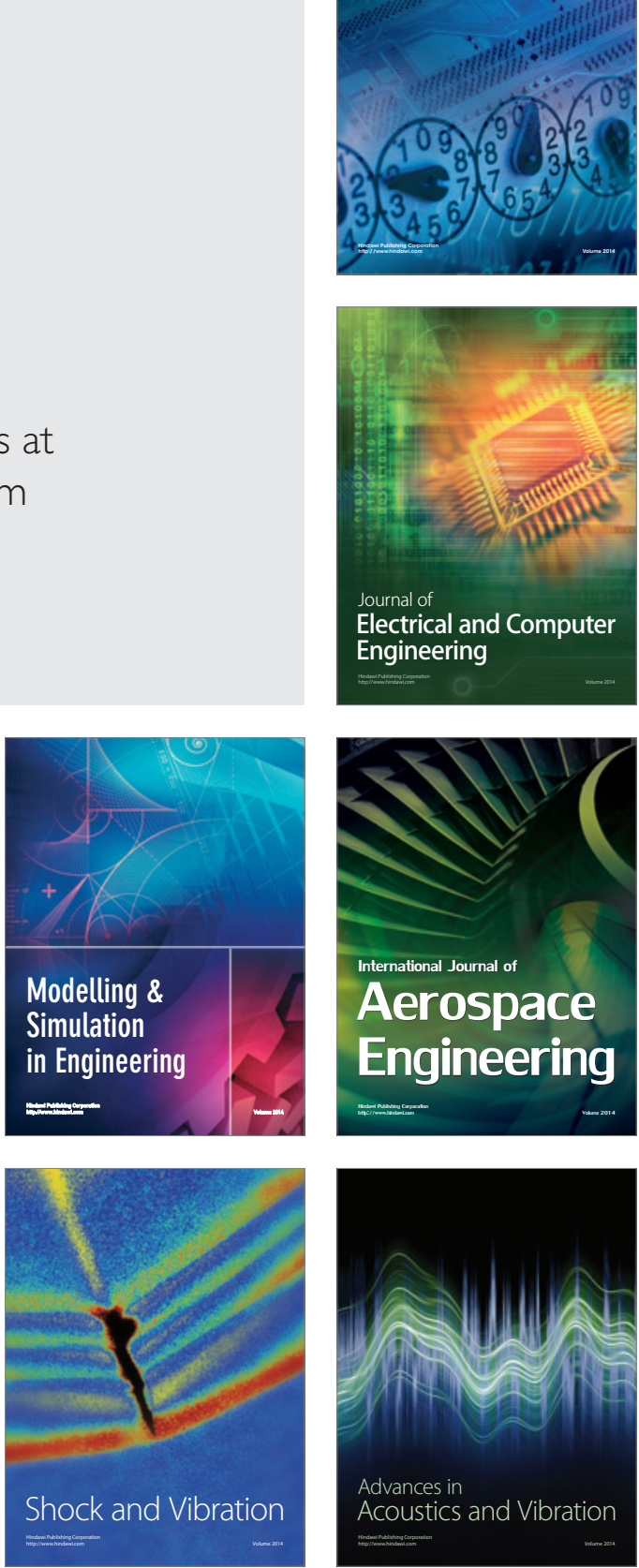